\title{
Astrocyte Specificity and Coverage of hGFAP- CreERT2 [Tg(GFAP-Cre/ERT2)13Kdmc] Mouse Line in Various Brain Regions
}

\author{
Yongmin Mason Park ${ }^{1,2,3 \dagger}$, Heejung Chun ${ }^{2,3 \dagger}$, Jeong-Im Shin ${ }^{1,2,3}$ and C. Justin Lee ${ }^{1,2,3 *}$ \\ ${ }^{1}$ Division of Bio-Medical Science \& Technology, Department of Neuroscience, KIST School, Korea University of Science and \\ Technology, Seoul 02792, ${ }^{2}$ Center for Glia-Neuron Interaction, Korea Institute of Science and Technology (KIST), Seoul 02792, \\ ${ }^{3}$ Center for Cognition and Sociality, Institute for Basic Science, Daejeon 34126, Korea
}

Astrocyte is the most abundant cell type in the central nervous system and its importance has been increasingly recognized in the brain pathophysiology. To study in vivo function of astrocyte, astrocyte-specific gene-targeting is regarded as a powerful approach. Especially, hGFAP-CreERT2, which expresses tamoxifen-inducible Cre recombinase under the human GFAP promoter, has been developed and characterized from several research groups. However, one of these mouse lines, [Tg(GFAP-Cre/ERT2)13Kdmc] from Ken McCarthy group has not been quantitatively analyzed, despite its frequent use. Here, we performed comprehensive characterization of this mouse line with quantitative analysis. By crossing this mouse line with Ai14 (RCL-tdTomato), a very sensitive Cre reporter mouse line, we visualized the Cre-expressing cells in various brain regions. For quantitative analysis, we immunostained S100 $\beta$ as an astrocytic marker and NeuN, tyrosine hydroxylase or calbindin as a neuronal marker in different brain regions. We calculated astrocyte specificity' as the proportion of co-labelled S100 $\beta$ and tdTomato positive cells in the total number of tdTomato positive cells and the astrocyte coverage' as the proportion of co-labelled $S 100 \beta$ and tdTomato positive cells in the total number of S100 $\beta$ positive cells. Interestingly, we found varying degree of astrocyte specificity and coverage in each brain region. In cortex, hypothalamus, substantia nigra pars compacta and cerebellar Purkinje layer, we observed high astrocyte specificity (over $89 \%$ ) and relatively high astrocyte coverage (over 70\%). In striatum, hippocampal CA1 layer, dentate gyrus and cerebellar granule layer, we observed high astrocyte specificity (over 80\%), but relative low astrocyte coverage (50-60\%). However, thalamus and amygdala showed low astrocyte specificity (about 65\%) and significant neuron specificity (over 30\%). This hGFAP-CreERT2 mouse line can be useful for genetic modulations of target gene either in gain-of-function or loss-of-function studies in the brain regions with high astrocyte specificity and coverage. However, the use of this mouse line should be restricted to gain-of-function studies in the brain regions with high astrocyte specificity but low coverage. In conclusion, hGFAP-CreERT2 mouse line could be a powerful tool for gene-targeting of astrocytes in cortex, striatum, hippocampus, hypothalamus, substantia nigra pars compacta and cerebellum, but not in thalamus and amygdala.

Key words: Astrocytes, Glial fibrillary astrocytic protein, Cre recombinase, Tamoxfien

Received November 30, 2018, Revised December 12,2018,

Accepted December 12, 2018

* To whom correspondence should be addressed.

INTRODUCTION

TEL: 82-42-878-9150, FAX: 82-42-878-9151

e-mail: cjl@ibs.re.kr

"These authors contributed equally to this work.

Astrocytes are increasingly recognized as an important cell type 
in the brain pathophysiology. Physiologically, astrocytes not only function at developmental stages by guiding neuronal migration [1] but also contribute to neuronal functions including taking up neurotransmitters through their transporters [2], sensing neuronal activity through many neurotransmitter receptors [3] and releasing gliotransmitters such as glutamate, GABA, ATP and D-serine [4-7]. Additionally, they also buffer extracellular potassium for proper neuronal firing [8]. At pathological conditions, including neurodegenerative disease, astrocytic functions are known to be altered [9]. For example, it was reported that glutamate transporter 1 (GLT1) levels are reduced [10] and tonic $\gamma$-aminobutyric acid (GABA) release is increased in animal models of Alzheimer's disease [11], which cause excitotoxicity and neuronal inhibition, respectively.

During these processes, astrocytic gene expression is also altered and involved in these functional alternation. For studying in vivo functions, astrocytic gene manipulation is required. For this, promoters of human glial fibrillary acidic protein (hGFAP), mouse aldehyde dehydrogenase family 1, member L1 (mALDH1L1) and human ALDH1L1 (hALDH1L1) were introduced to modulate gene expression in astrocytes by expressing Cre recombinase (Cre) cell type-specific manner through transgenic mouse or virus injection [12-14]. However, these promoters showed leaky Cre expression in other brain cell types such as neuron, microglia or oligodendrocytes $[13,15]$. Therefore, their astrocyte specificity has been questioned. In particular, it has been reported that transient GFAP expression in neuronal progenitor cells causes significant proportion of neuronal Cre expression in hGFAP-Cre mouse line [15]. Therefore, utilizing hGFAP-Cre mouse line is not suitable for targeting mature astrocytes. Based on this issue, inducible Cre driver transgenic mouse lines such as hGFAP-CreERT2, GLASTCreERT2 and ALDH1L1-CreERT2 have been developed [1621]. However, GLAST-CreERT2 also shows a significant neuronal expression, whereas ALDH1L1-CreERT2 does not [20]. Although $\mathrm{m} A L D H 1 L 1$ promoter has high astrocyte specificity in the brain, it has significant limitations to utilize in brain-specific manner by the fact that ALDH1L1 mRNA is also highly expressed in other parts of the body [22]. Due to these limitations, until now there is no perfect option for genetically targeting astrocyte in the brain.

hGFAP-CreERT2 mouse lines, which are the most popular transgenic mouse line for genetically targeting astrocyte, were made by several groups $[16-18,21]$. Two mouse lines which were initially developed each from Vaccarino group [Tg(GFAP-cre/ ERT2) 505Fmv/J] (MGI: 3774167), Kirchhoff group [Tg(GFAPcre/ERT2) 1Fki] (MGI: 4418665), and Baker group were very well characterized with detailed quantitative analysis of astrocyte specificity. However, the other mouse line which is from Ken Mc-
Carthy group [Tg(GFAP-cre/ERT2) 13Kdmc], (MGI:3712447) has not been well characterized in various brain regions, despite its frequent use with over 75 citations of the original paper [23-25]. In this regard, it is necessary to characterize the cell types and its exact proportion in various brain regions for accurate interpretation of results.

Based on this need, we set out to characterize [Tg(GFAP-cre/ ERT2) $13 \mathrm{Kdmc}$, which we refer to as hGFAP-CreERT2, for assessing its efficiency of targeting astrocyte in various brain regions. To quantitatively analyze Cre expression pattern by crossing with Ail 4 mouse line whose floxed tdTomato fluorescence gene can be sensitively and brightly activated by Cre. In this double transgenic mouse line, we analyzed the population of tdTomato positive cells to examine the astrocyte specificity', indicating the proportion of astrocyte in the total number of Cre expressing cells, and astrocyte coverage', indicating the proportion of Cre expressing cells in the total number of astrocytes, of the hGFAP-CreERT2 mouse line depending on brain regions.

\section{MATERIALS AND METHODS}

\section{Animals}

To generate reporter mice for visualizing Cre expression, we made double transgenic mouse line in C57BL/6J strain by crossing Ail4 (Rosa-CAG-LSL-tdTomato-WPRE::deltaNeo) mouse with hGFAP-CreERT2 mouse (hGFAP-CreERT2×Ai14). The mice have been bred in suitable space mice cage (Thoren, Hazleton, USA) with freely accessing to food and water and kept on a 12-hour-light-dark cycle. All experimental steps described in this paper were performed in accordance with the institutional guidelines for experimental animal care and use of the Korea Institute of Science and Technology (KIST; Seoul, Korea).

\section{Genotyping}

Mouse tails were digested overnight at $60^{\circ} \mathrm{C}$, using $1 \mathrm{mg} / \mathrm{ml}$ Proteinase K (21560025-2, bioWORLD, Dublin, USA) in lysis reagent for genotyping (102-T, Viagenbiotech, Los Angeles, USA). On the following day, after inactivating the protein kinase $\mathrm{K}$, the supernatant containing DNA was used for genotyping. The PCR reaction mixture contained Taq DNA Polymerase, $\mathrm{MgCl}_{2}, \mathrm{dNTP}$ mix (\# QM13531, Bioquest, Seoul, Korea) and $1 \mu$ genomic DNA template, $0.1 \mu \mathrm{M}$ forward and reverse primer pairs and $\mathrm{dd}_{2} \mathrm{O}$. PCR was performed using the specific primer pairs as the detailed protocol is described in Fig. 1B. The reaction products were run on 1.5\% agarose gels (HB0100500, E\&S Bio Electronics, Daejeon, Korea) in TAE buffer (40 mM Tris: $\mathrm{pH}$ 7.6, $20 \mathrm{mM}$ acetic acid and $1 \mathrm{mM}$ EDTA) at $100 \mathrm{~V}$ and visualized using Nobel view (NOV001, 

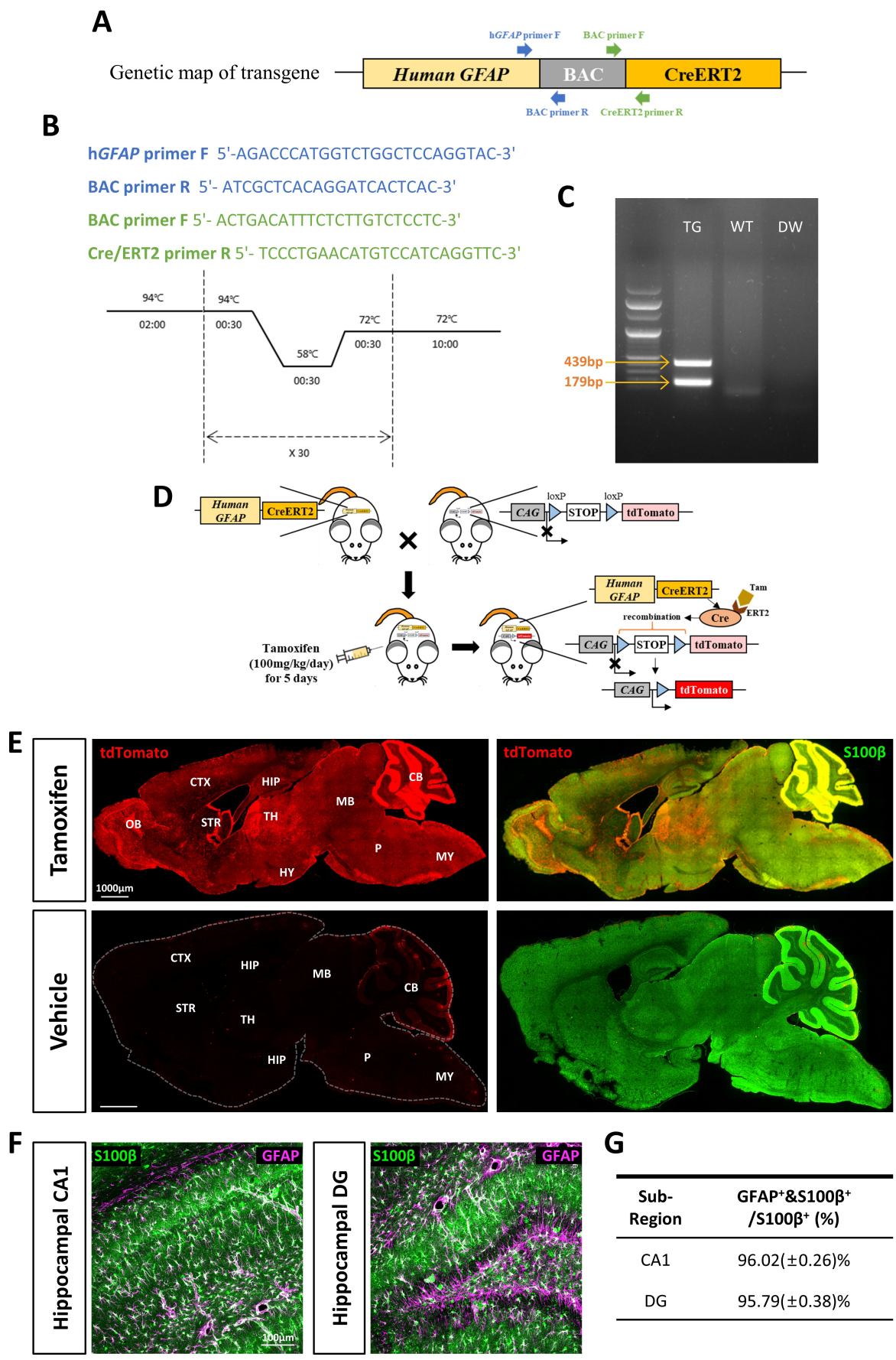

Fig. 1. Genetic confimation of hGFAP-CreERT2 mouse line and its tamoxifen inducible Cre expression. (A) Genetic map of hGFAP-CreERT2. (B) The primer sequences and PCR protocol of hGFAP-CreERT2 transgenic mice. (C) PCR results of genotyping; TG: Transgenic mouse, WT: Wild-Type, DW: $\mathrm{dd}_{2} \mathrm{O}$ (D) Schematic diagram of generation of double transgenic mouse. (E) Sagittal brain section images from tamoxifen or vehicle (sunflower oil) treated hGFAP-CreERT2 mouse. Red fluorescence indicates tdTomato, and green fluorescence indicates S100 $\beta$. OB=Olfactory bulb, CTX=Cortex, $\mathrm{STR}=$ Striatum, HIP=Hippocampus, TH=thalamus, $\mathrm{HY}=$ Hypothalamus, $\mathrm{CB}=$ Cerebellum, MB=Midbrain, $\mathrm{P}=\mathrm{Pons}$, and MY=Medulla. (F) S100 $\beta$ and GFAP localization images of hippocampal CA1 layer (CA1, left) and dentate gyrus (DG, middle). Green fluorescence indicates S100 $\beta$, and magenta fluorescence indicates GFAP. (G) Summary table of co-localization ratio of S100 $\beta^{+}$\& GFAP ${ }^{+}$cells among S100 $\beta^{+}$cells in each images, $(\mathrm{CA1}$ : $\mathrm{n}=4$ sections, DG: $\mathrm{n}=2$ sections from 2 mice) $($ Mean \pm SEM $)$. 
Noble Bio, Hwaseong-si, Gyeonggi-do, Korea).

\section{Tamoxifen administration}

Stock solution of tamoxifen was made at concentration of $20 \mathrm{mg} /$ $\mathrm{ml}$ in sunflower seed oil (Sigma, St.Louis, USA) mixture with 1/10 ethanol (64-17-5, Millipore, Burlington, USA). $100 \mu \mathrm{l}$ of tamoxifen were administered into each hGFAP-CreERT2×Ai14 double transgenic mice $(8 \sim 12$ weeks, male and female) by intraperitoneal (i.p.) injection per day for consecutive 5 days.

\section{Immunohistochemistry}

Mice were anesthetized with $2 \%$ avertin $(20 \mu \mathrm{l} / \mathrm{g}$, T48402, Sigma) by intraperitoneal injection and perfused with $4 \%$ paraformaldehyde accompanied by $0.1 \mathrm{M}$ phosphate buffered saline (PBS) at room temperature. Extracted whole brains were post-fixed in $4 \%$ paraformaldehyde at $4^{\circ} \mathrm{C}$ overnight. After that, post-fixed brains were incubated in $30 \%$ sucrose at $4^{\circ} \mathrm{C}$ for more than 24 hours. Both sagittal and coronal sections were sliced with $30 \mu \mathrm{m}$ thickness in cryostat microtome (Thermo Scientific, Waltham, USA). Sliced brain sections were washed three times with PBS and blocked with blocking solution (0.3\% Triton X-100 (X-100, Sigma), 2\% donkey serum (GTX27475, Genetex, Irvine, USA) and $2 \%$ goat serum (ab7481, Abcam, Cambridge, UK) in 0.1 M PBS for 1.5 hours. And then, these sections were incubated in suitable primary antibodies (rabbit anti-S100 $\beta$ antibody (ab41548, Abcam) and mouse anti-NeuN antibody (MAB377, Abcam) or chicken anti-tyrosine hydroxylase (P40101-0 TH, Abcam) or mouse antiCalbindin antibody (C9848, Sigma) mixture with shaking at $4^{\circ} \mathrm{C}$ overnight. After overnight incubation, brain sections were washed three times with PBS and incubated in matched secondary antibodies (Jackson, Bar Harbor, USA), they were also stained with 4',6'-diamidino-2-phenylindole (DAPI, 62248, Pierce, Waltham, USA). Every sagittal sections' fluorescence images were taken by Operetta ${ }^{\circledR}$ High Contents Screening System. On the other hand, every coronal sections' fluorescence images were taken by Nikon A1 confocal microscope, and $30 \mu \mathrm{m} Z$ stack images in 2- $\mu \mathrm{m}$ steps were processed by utilizing NIS-Elements (Nikon, Minato ku, Japan) software. Quantitative analysis method is described below paragraph. Reference atlases of mouse brain in the figure were obtained from Allen Brain Atlas (Reference Atlas, Version 2 (2011)) [26].

\section{Quantitative analysis}

Coronal sections' fluorescence images were quantitatively analyzed by utilizing ImageJ (NIH) software. We identified astrocyte (also Bergmann glia) or neuron based on their morphology and co-localization with suitable each cell-type specific markers as we described above. After that, we counted and calculated Cre activated cell portion of each neurons and astrocytes which are co-localized with Cre mediated tdTomato fluorescence positive cell based on DAPI positive cell. Moreover, we defined astrocyte specificity' and 'astrocyte coverage. The former indicates the percentage of S100 $\beta$ \& tdTomato co-positive cells over total tdTomato positive cells, and the latter indicates that of $\mathrm{S} 100 \beta$ \& tdTomato copositive cells over total $S 100 \beta$ positive cells.

\section{RESULTS}

\section{Genotypic and phenotypic confirmation of hGFAP-Cre- ERT2 mouse line}

According to the original description of hGFAP-CreERT2 mouse line, this mouse line was generated by micro injection of plasmid form of the transgene $(2.2 \mathrm{~kb}$ human GFAP promoter and CreERT2) into mouse embryonic stem cell [16]. For genetic confirmation, we designed two unique sets of primers, each targeting the promoter and transgene, and performed polymerase chain reaction (PCR) of tail biopsy genomic DNA (Fig. 1A and B). Gel electrophoresis of the PCR products showed two bands at $439 \mathrm{bp}$ and 179 bp (Fig. 1C), indicating hGFAP promoter and CreERT2, respectively.

To characterize cell type specificity of tamoxifen-inducible Creexpressing cells in hGFAP-CreERT $2 \times$ Ail 14 double transgenic mouse line, we administered tamoxifen or sunflower oil in these mice by i.p. injection for five consecutive days (Fig. 1D). Upon tamoxifen administration, we observed the tdTomato fluorescence in Cre-expressing cells. To scan and validate the Cre-expressing cells as an astrocyte, we performed immunohistochemistry by using an antibody against $S 100 \beta$ as an astrocytic marker in sagittal brain sections (Fig. 1E, top). These sections showed ubiquitous Cre expression in all brain regions (Fig. 1E, top, left). Moreover, most of these cells showed co-localized immunoreactivity with S100 $\beta$ (Fig. 1E, top, right). In contrast, the control mouse (hGFAPCreERT2 $\times$ Ai14) with vehicle treatment (sunflower oil) showed minimal tdTomato fluorescence, indicating that there is virtually no leaky expression of Cre without tamoxifen (Fig. 1E, bottom, left). Furthermore, tdTomato fluorescence showed varying degree of co-localization with $S 100 \beta$ in various brain regions, as evidenced by intensity of yellow color (Fig. 1E, top, right). For example, in cortex, hippocampus and cerebellum, tdTomato expressing cells were majorly merged with $\$ 100 \beta$ showing intense yellow color, whereas in thalamus tdTomato expressing cells were rarely merged with $\mathrm{S} 100 \beta$ showing segregated two distinct colors (Fig. 1E, top, right). These results imply that the degree of astrocyte specificity is different in various brain regions. 
These regional differences in the degree of astrocyte specificity could be originated from either the lack of specificity of $S 100 \beta$ antibody to astrocytes or the region-dependent differential activity of human GFAP promoter in non-astrocytes. In fact, it has been reported previously that some $S 100 \beta$ antibodies labels oligodendrocyte progenitor cells (OPCs) in the developing brain [27-29]. However, there has been no information on the proportion of S100 $\beta$-positive astrocytes in adult brain. Therefore, to directly determine what proportions of S100 $\beta$-positive cells are GFAP-positive astrocytes or GFAP-negative (non-astrocytic, possibly OPCs) cells, we co-immunostained the adult mouse brain with GFAP and S100 $\beta$ antibodies and observed that about $96 \%$ of S100 $\beta$-positive cells were GFAP-positive astrocytes in hippocampal CA1 and DG regions (Fig. 1F and 1G). These results indicate that the $S 100 \beta$ antibody is an excellent astrocyte marker in adult brain. Therefore, by using this $S 100 \beta$ antibody as a reliable astrocyte marker, we quantitatively analyzed cell type-specific expression of Cre in various brain regions where astrocytic functions are extensively researched.

\section{In cortex (somatomotor area) and striatum (caudoputa- men), hGFAP-CreERT2 mouse shows high astrocyte speci- ficity but differential coverage}

To investigate tamoxifen-inducible Cre-expressing cells in cortex, we performed immunohistochemistry by utilizing $S 100 \beta$ as an astrocytic marker rather than GFAP, which is known to be differentially expressed in various brain regions [30]. We used NeuN (Neuronal nuclear antigen) as a neuronal marker for quantitative analysis (Fig. 2A). We found that tdTomato expressing cells in each image were predominantly co-localized with $\mathrm{S} 100 \beta$ in cortex (Fig. 2B and C, green, $87.37 \pm 1.45 \%$ ). However, these tdTomato expressing cells were rarely co-localized with $\mathrm{NeuN}$ (Fig. 2B and C, cyan, $8.04 \pm 1.03 \%$ ). Based on these high magnification images, we calculated 'astrocyte specificity' which is the ratio of the total counted number of tdTomato and $S 100 \beta$ double positive $\left(\right.$ tdTomato $\left.^{+} \& S 100 \beta^{+}\right)$cells to that of tdTomato positive (tdTomato $^{+}$) cells. Moreover, we also calculated astrocyte coverage' which is the ratio of the total counted number of $\operatorname{tdTomato}^{+} \& S 100 \beta^{+}$ cells to that of $S 100 \beta^{+}$cells. Based on these calculations, astrocyte specificity was $87.69 \%$ and coverage was $74.13 \%$ in cortex (Fig. 3D and E). These results indicate that hGFAP-CreERT2 mouse line has high astrocyte specificity with over $80 \%$ of specificity and high astrocyte coverage with over $70 \%$ of coverage in cortex.

In striatum (caudoputamen) (Fig. 3A), tdTomato expressing cells in each image also showed pre-dominant $\mathrm{S} 100 \beta$ co-localization which was comparable or higher than that of cortex (Fig. $3 \mathrm{~B}$ and C, green, $90.04 \pm 3.42 \%$ ) and rare NeuN co-localization (Fig. 3B and C, cyan, $2.71 \pm 1.25 \%$ ). Astrocyte specificity was $89.02 \%$ and coverage was $50.69 \%$. (Fig. 3D and E). These results indicate that hGFAPCreERT2 mouse line has high astrocyte specificity with over $80 \%$ of specificity, but low astrocyte coverage with under $70 \%$ of coverage in striatum.

\section{In hippocampus, hGFAP-CreERT2 mouse shows high astro- cyte specificity but low coverage}

Next, we examined hippocampal CA1 layer (CA1) and Dentate Gyrus (DG). In hippocampal CA1 (Fig. 4A), tdTomato expressing cells in each image were highly co-localized with S100 $\beta$ (Fig. $4 \mathrm{~B}$ and D, green, $92.61 \pm 1.49 \%$ ) but not with NeuN (Fig. 4B and D, cyan, $5.21 \pm 2.10 \%$ ). Astrocyte specificity was $92.83 \%$ and coverage was $56.28 \%$ (Fig. 4E and F, left).

In DG area (Fig. 4B), tdTomato expressing cells in each image were highly co-localized with $\mathrm{S} 100 \beta$ (Fig. 4C and D, green, $85.91 \pm 1.08 \%$ ) than that of NeuN (Fig. 4C and D, cyan, $10.59 \pm 1.13 \%)$. Although, a few tdTomato expressing cells were found in subgranular zone where GFAP positive neuronal progenitor cells are known to be present [31], the total number was negligible $(<5 \%)$. Astrocyte specificity was $86.13 \%$ and coverage was $61.44 \%$ (Fig. 4E and F, right). These results indicate that hGFAPCreERT2 mouse line has high astrocyte specificity with over $80 \%$ of specificity, but relatively low astrocyte coverage with under $70 \%$ of coverage in both hippocampus sub-regions.

\section{In LHA and SNpC, hGFAP-CreERT2 mouse shows high as- trocyte specificity and coverage}

We further tested Cre expression pattern in lateral hypothalamus (LHA) (Fig. 5A). In LHA, tdTomato expressing cells were majorly S100 $\beta^{+}$in each image (Fig. $5 B$ and C, green, $92.05 \pm 2.39 \%$ ), but $\mathrm{NeuN}^{+}$cells were little (Fig. 5B and C, cyan, 5.48 $\pm 2.61 \%$ ). Astrocyte specificity was $92.20 \%$ and coverage was $79.24 \%$ in LHA (Fig. 5D and E). These results indicate hGFAP-CreERT2 mouse line has high astrocyte specificity with over $80 \%$ of specificity and high astrocyte coverage with over $70 \%$ of coverage in LHA.

Similar with LHA, we also tested in substantia nigra pars compacta (SNpC) by using S100 $\beta$ and tyrosine hydroxylase (TH) which marks dopaminergic neuron (Fig. 6A). Almost all of tdTomato expressing cells in $\mathrm{SNpC}$ were also $\mathrm{S} 100 \beta^{+}$in each image (Fig. 6B and $\mathrm{C}$, green, $97.74 \pm 0.59 \%$ ), but no $\mathrm{TH}^{+}$cells (Fig. 6B and C, cyan, $0.00 \%$ ). In $\mathrm{SNpC}$, astrocyte specificity was $97.64 \%$ and coverage was $72.94 \%$ (Fig. 6D and E). These results indicate that hGFAPCreERT2 mouse line has the highest astrocyte specificity and high astrocyte coverage with over $70 \%$ of coverage in $\mathrm{SNpC}$. 

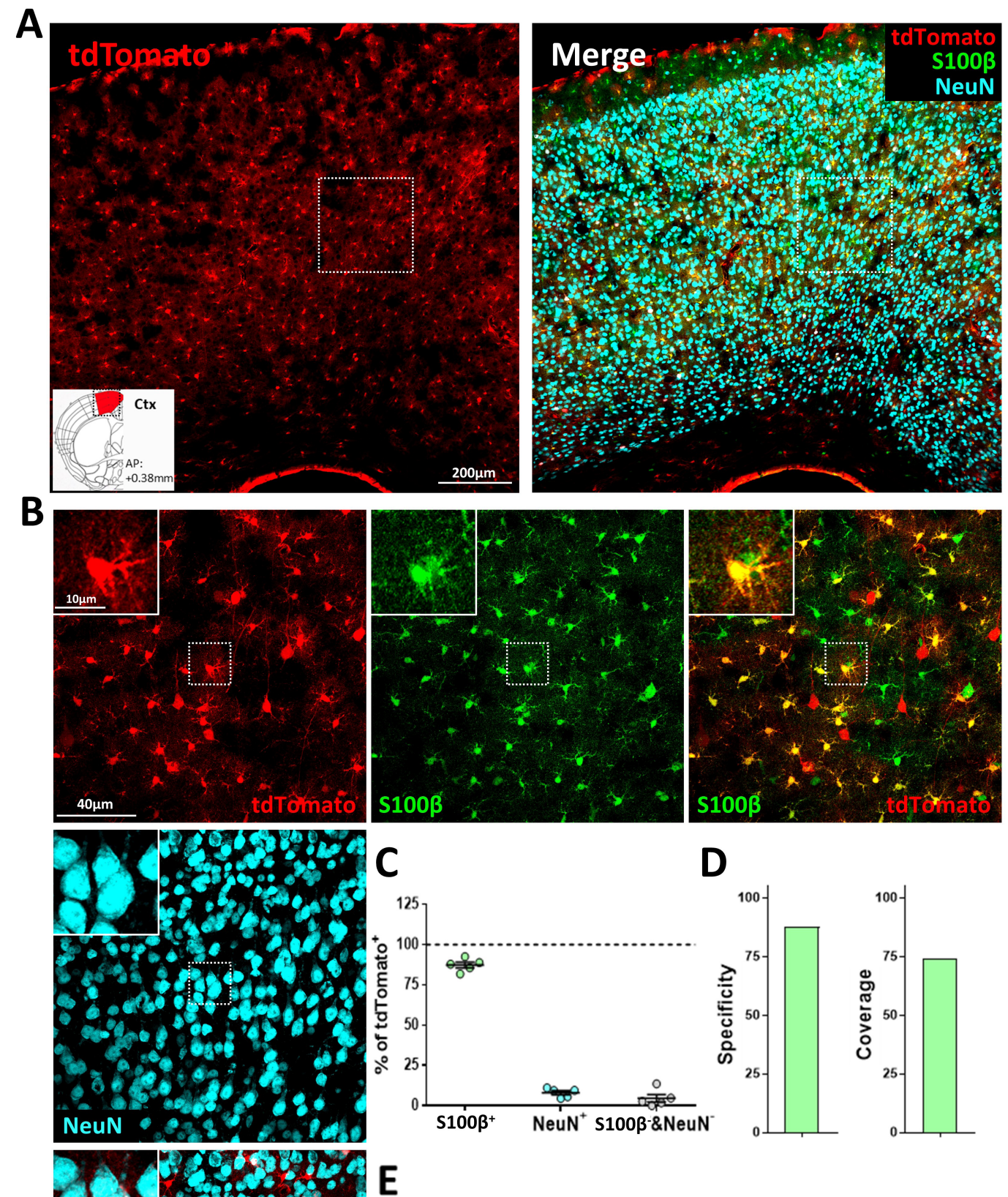

D
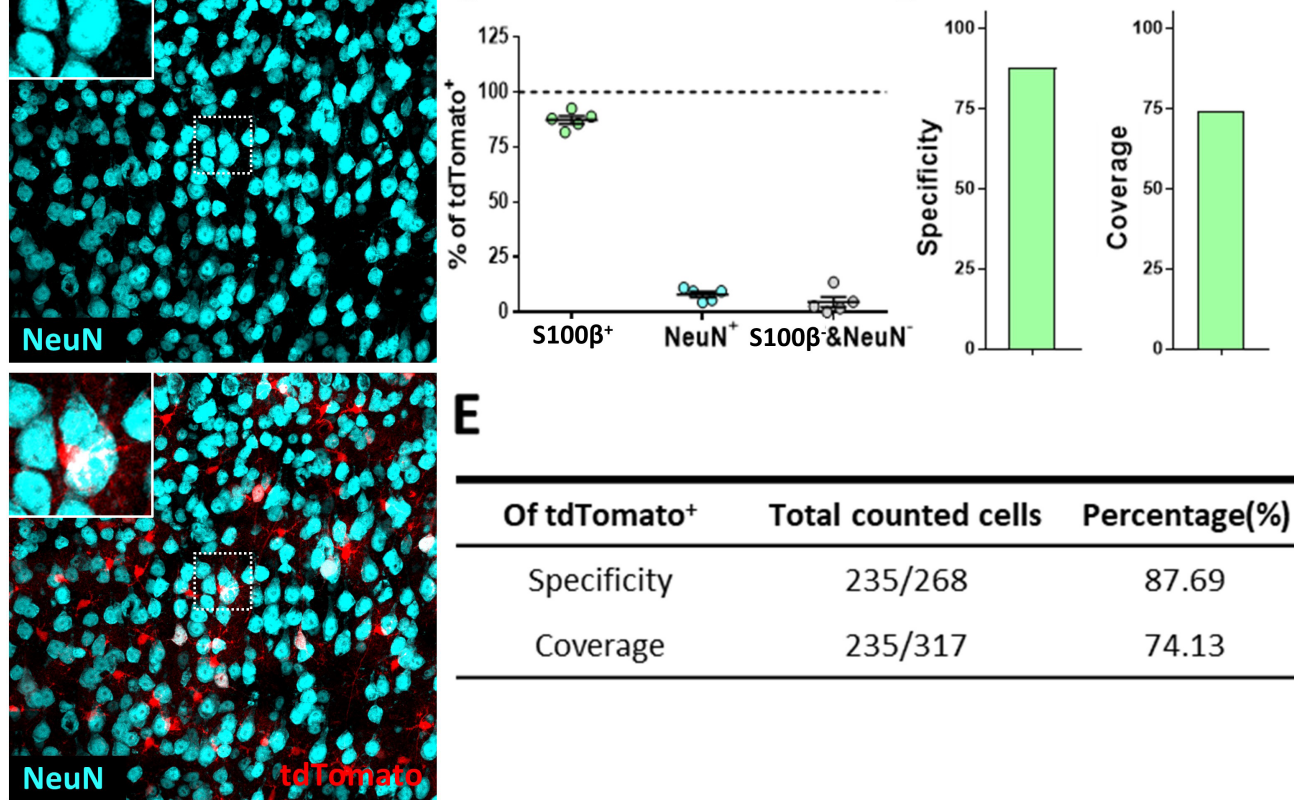

\begin{tabular}{ccc}
\hline Of tdTomato $^{+}$ & Total counted cells & Percentage(\%) \\
\hline Specificity & $235 / 268$ & 87.69 \\
Coverage & $235 / 317$ & 74.13 \\
\hline
\end{tabular}

Fig. 2. In cortex (somatomotor area), tdTomato expression driven by hGFAP-CreERT2. (A) Low magnification images of cortex. Each color represents as following description. Green=S100ß; Red=tdTomato; Cyan=NeuN. (B) High magnification images from the box in (A). (C) Quantification of tdTomato ${ }^{+}$population with $\mathrm{S} 100 \beta^{+}$(green), $\mathrm{NeuN}^{+}$(cyan) and $\mathrm{S} 100 \beta \& \mathrm{NeuN}^{-}$(gray) cells in cortex (Mean $\pm \mathrm{SEM}, \mathrm{n}=5$ sections from 3 mice). (D) Summary graph of astrocyte specificity $\left(\right.$ tdTomato $^{+} \& S 100 \beta^{+} /$tdTomato $\left.^{+}\right)$and coverage $\left(\operatorname{td} \operatorname{Tomato}^{+} \& S 100 \beta^{+} / S 100 \beta^{+}\right)$. (E) Summary table of above analysis. 

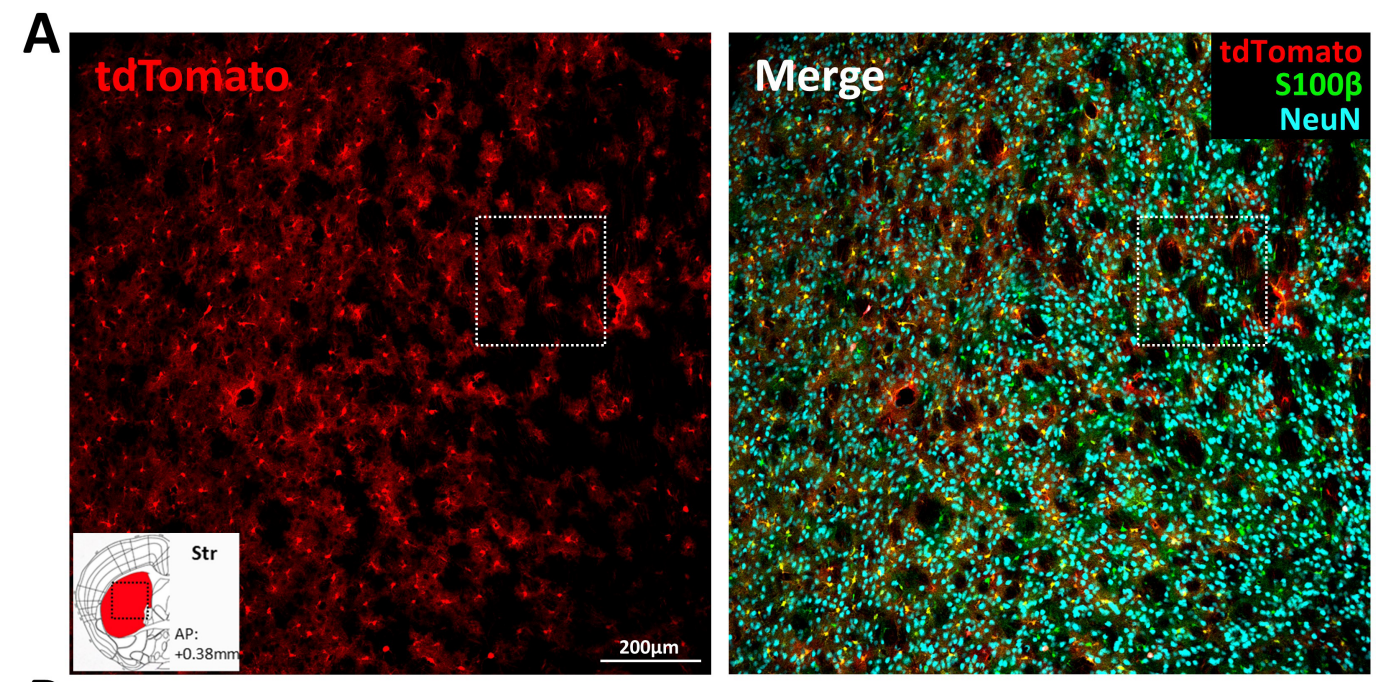

B
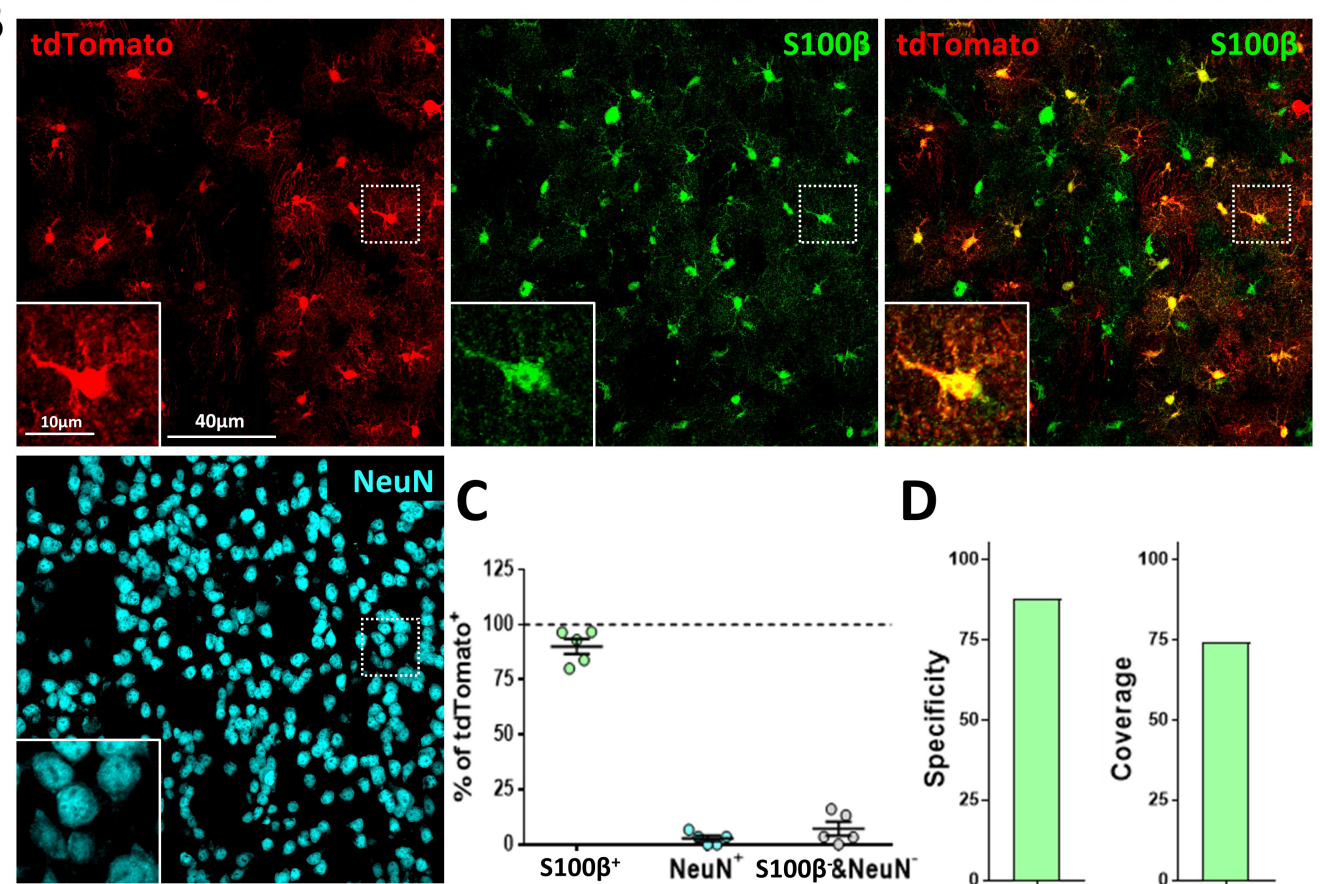

D
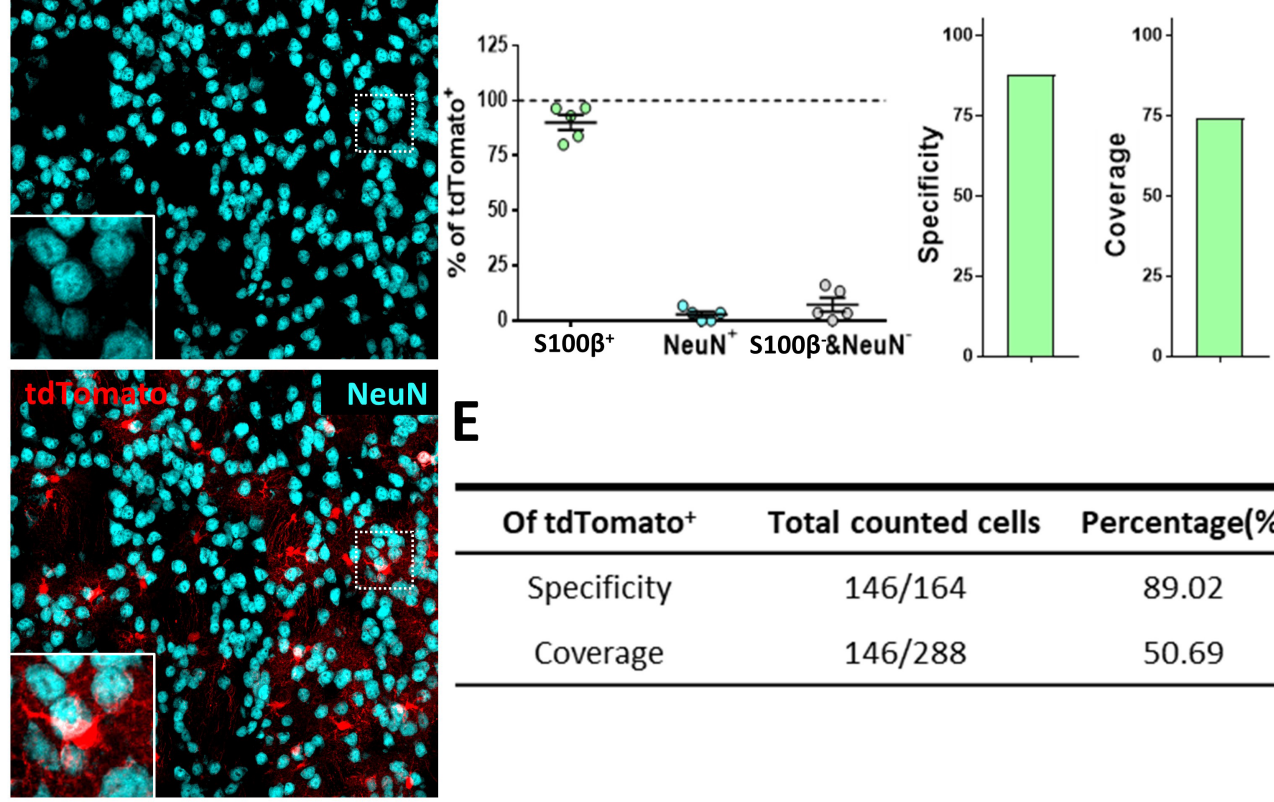

$E$

\begin{tabular}{ccc}
\hline Of tdTomato $^{+}$ & Total counted cells & Percentage(\%) \\
\hline Specificity & $146 / 164$ & 89.02 \\
Coverage & $146 / 288$ & 50.69 \\
\hline
\end{tabular}

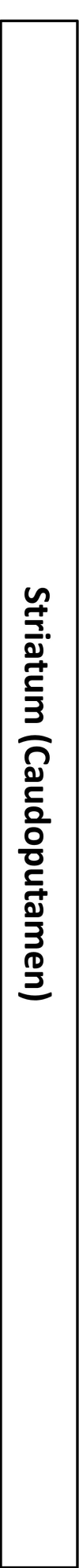

Fig. 3. In striatum (Caudoputamen), tdTomato expression driven by hGFAP-CreERT2. (A) Low magnification images of striatum. Each color represents as following description. Green=S100ß; Red=tdTomato; Cyan=NeuN. (B) High magnification images from the box in (A). (C) Quantification of tdTomato $^{+}$population with $\mathrm{S}_{100} \beta^{+}$(green), $\mathrm{NeuN}^{+}$(cyan) and $\mathrm{S} 100 \beta$ \&NeuN (gray) cells in striatum (Mean $\pm \mathrm{SEM}, \mathrm{n}=5$ sections from 3 mice). (D) Summary graph of astrocyte specificity $\left(\mathrm{tdTomato}{ }^{+} \& \mathrm{~S} 100 \beta^{+} / \mathrm{tdTomato}^{+}\right)$and coverage $\left(\mathrm{tdTomato}{ }^{+} \& \mathrm{~S} 100 \beta^{+} / \mathrm{S} 100 \beta^{+}\right)$. (E) Summary table of above analysis. 


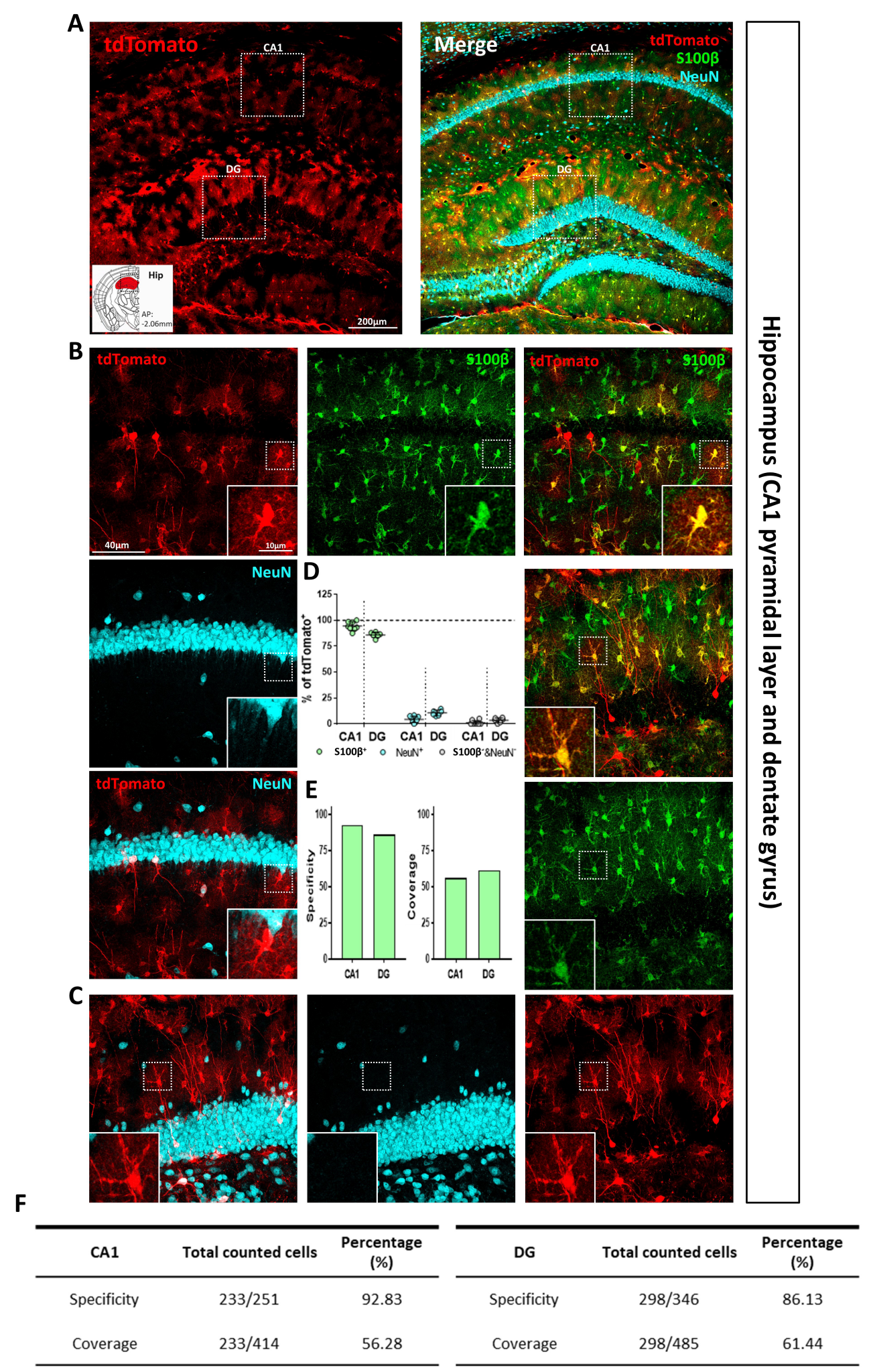

Fig. 4. In hippocampal CA1 layer (CA1) and Dentate gyrus (DG), tdTomato expression driven by hGFAP-CreERT2. (A) Low magnification images of hippocampal CA1 (left) and DG (right). Each color represents as following description. Green=S100ß; Red=tdTomato; Cyan=NeuN. (B) High magnification images from the box in CA1. (C) High magnification images from the box in DG. (D) Quantification of tdTomato ${ }^{+}$population with S100 $\beta^{+}$(green), $\mathrm{NeuN}^{+}$(cyan) and S100 $\beta$ \&NeuN (gray) cells in hippocampal CA1 and DG (Mean \pm SEM, $\mathrm{n}=6$ sections from 3 mice). (E) Summary graph of astrocyte specificity (tdTomato ${ }^{+} \& S 100 \beta^{+} /$tdTomato $\left.^{+}\right)$and coverage (tdTomato $\left.{ }^{+} \& S 100 \beta^{+} / \mathrm{S} 100 \beta^{+}\right)$from each region. (F) Summary table of above analysis $(\mathrm{CA} 1$ : left, DG: right). 

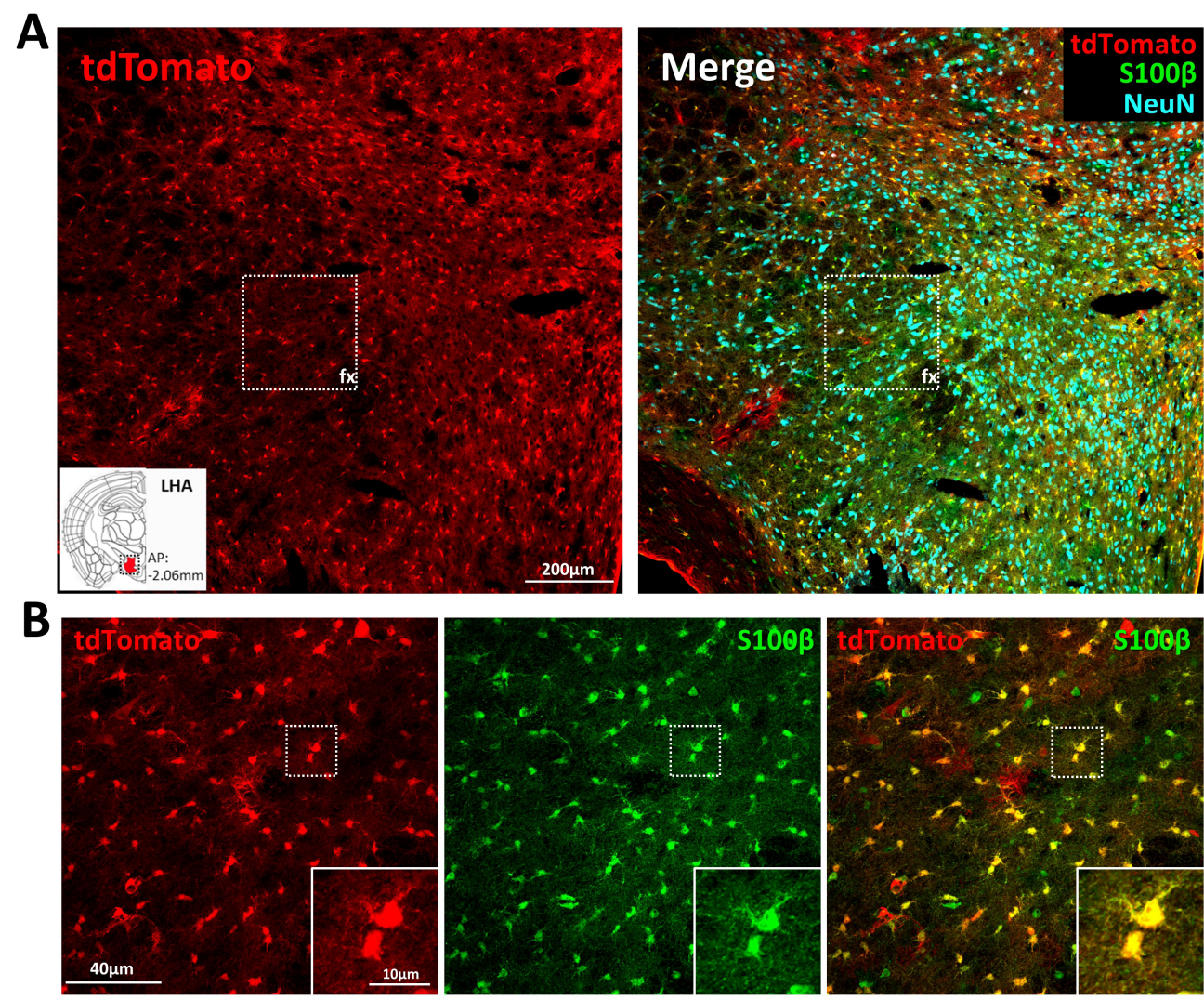
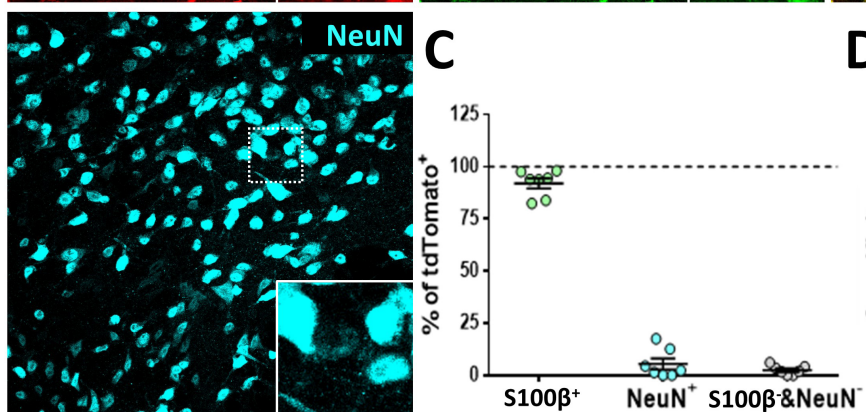

D
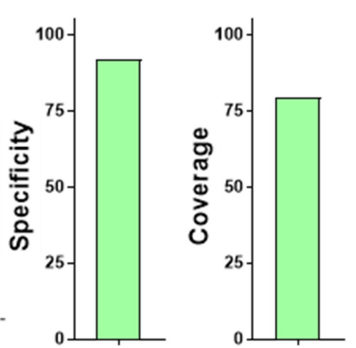

$E$

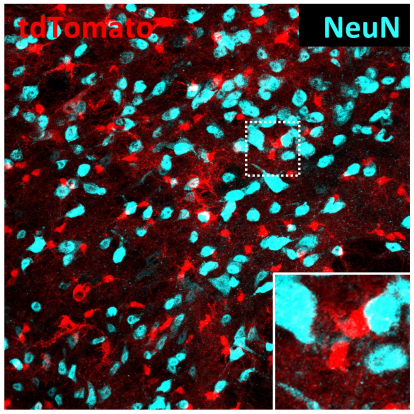

\begin{tabular}{ccc}
\hline Of tdTomato $^{+}$ & Total counted cells & Percentage(\%) \\
\hline Specificity & $603 / 654$ & 92.20 \\
Coverage & $603 / 761$ & 79.24 \\
\hline
\end{tabular}

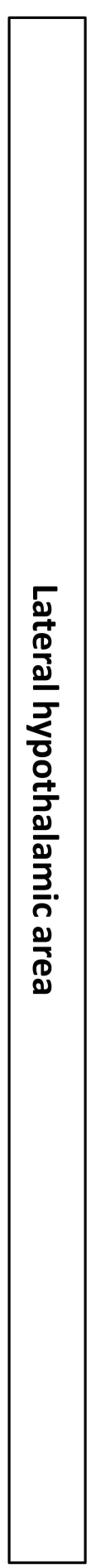

Fig. 5. In Lateral hypothalamic area (LHA), tdTomato expression driven by hGFAP-CreERT2. (A) Low magnification images of LHA. Each color represents as following description. Green=S100 $\beta$; Red=tdTomato; Cyan=NeuN. (B) High magnification images from the box in (A). (C) Quantification of tdTomato ${ }^{+}$population with $\mathrm{S}_{100 \beta^{+}}$(green), $\mathrm{NeuN}^{+}$(cyan) and $\mathrm{S} 100 \beta^{-} \& \mathrm{NeuN}^{-}$(gray) cells in LHA (Mean $\pm \mathrm{SEM}, \mathrm{n}=7$ sections from 3 mice). (D) Summary graph of astrocyte specificity $\left(\right.$ tdTomato $^{+} \& S 100 \beta^{+} /$tdTomato $^{+}$) and coverage $\left(\right.$tdTomato $\left.^{+} \& S 100 \beta^{+} / \mathrm{S} 100 \beta^{+}\right)$. (E) Summary table of above analysis. 

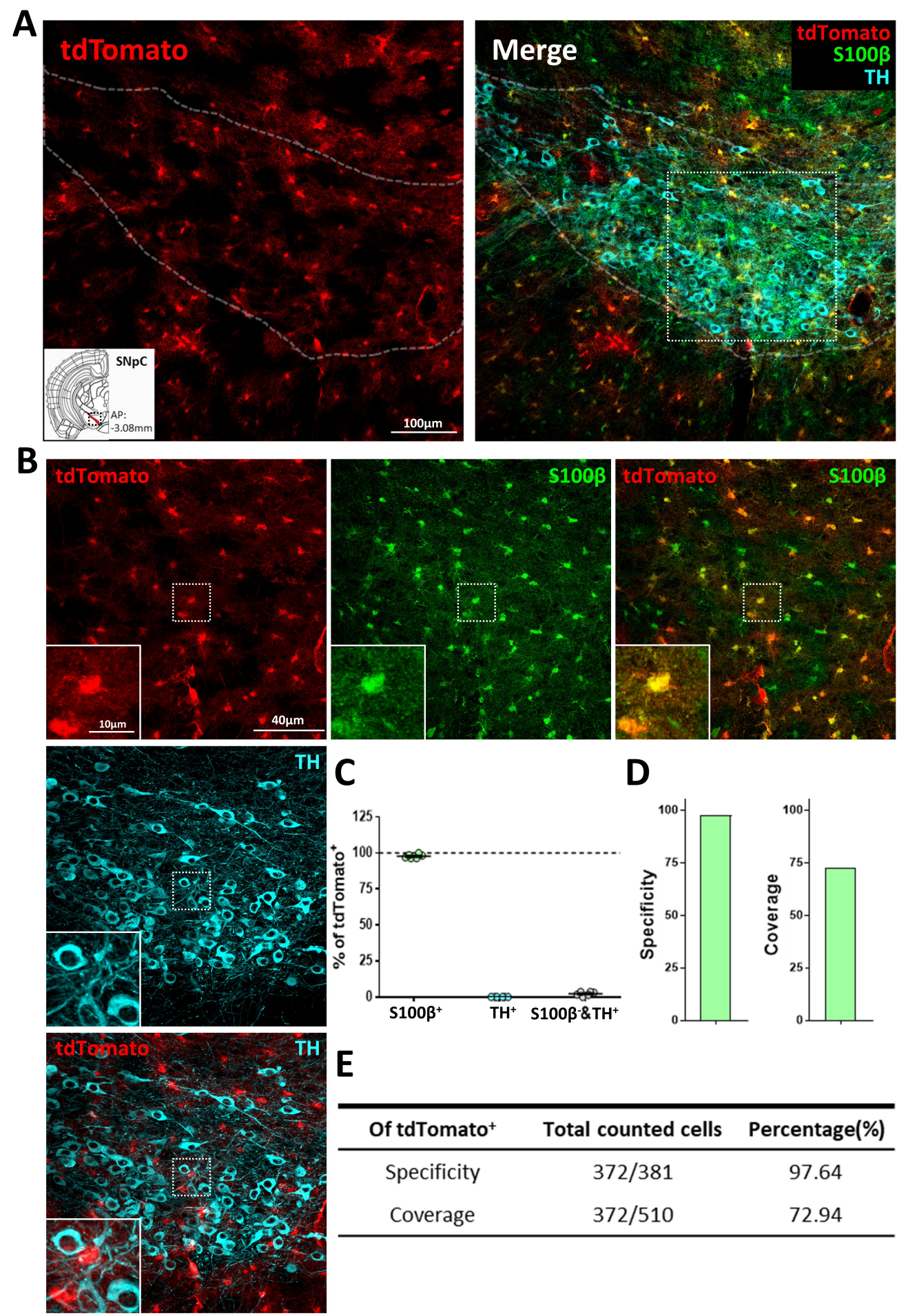

\section{E}

\begin{tabular}{ccc}
\hline Of tdTomato $^{+}$ & Total counted cells & Percentage(\%) \\
\hline Specificity & $372 / 381$ & 97.64 \\
Coverage & $372 / 510$ & 72.94 \\
\hline
\end{tabular}

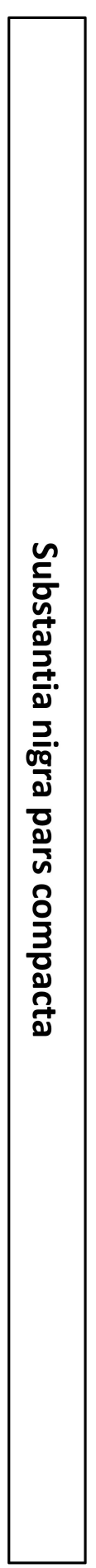

Fig. 6. In substantia nigra pars compacta ( $\mathrm{SNpC}$ ), tdTomato expression driven by hGFAP-CreERT2. (A) Low magnification images of SNpC. Each color represents as following description. Green=S100ß; Red=tdTomato; Cyan=TH. (B) High magnification images from the box in (A). (C) Quantification of tdTomato ${ }^{+}$population with S100 $\beta^{+}$(green), $\mathrm{TH}^{+}$(cyan) and S100 $\beta \& \mathrm{TH}^{-}$(gray) cells in SNpC (Mean $\pm \mathrm{SEM}, \mathrm{n}=6$ sections from 3 mice). (D) Summary graph of astrocyte specificity $\left(\right.$ tdTomato $^{+} \& S 100 \beta^{+} /$tdTomato $\left.^{+}\right)$and coverage $\left(\right.$tdTomato $\left.{ }^{+} \& S 100 \beta^{+} / S 100 \beta^{+}\right)$. (E) Summary table of above analysis. 


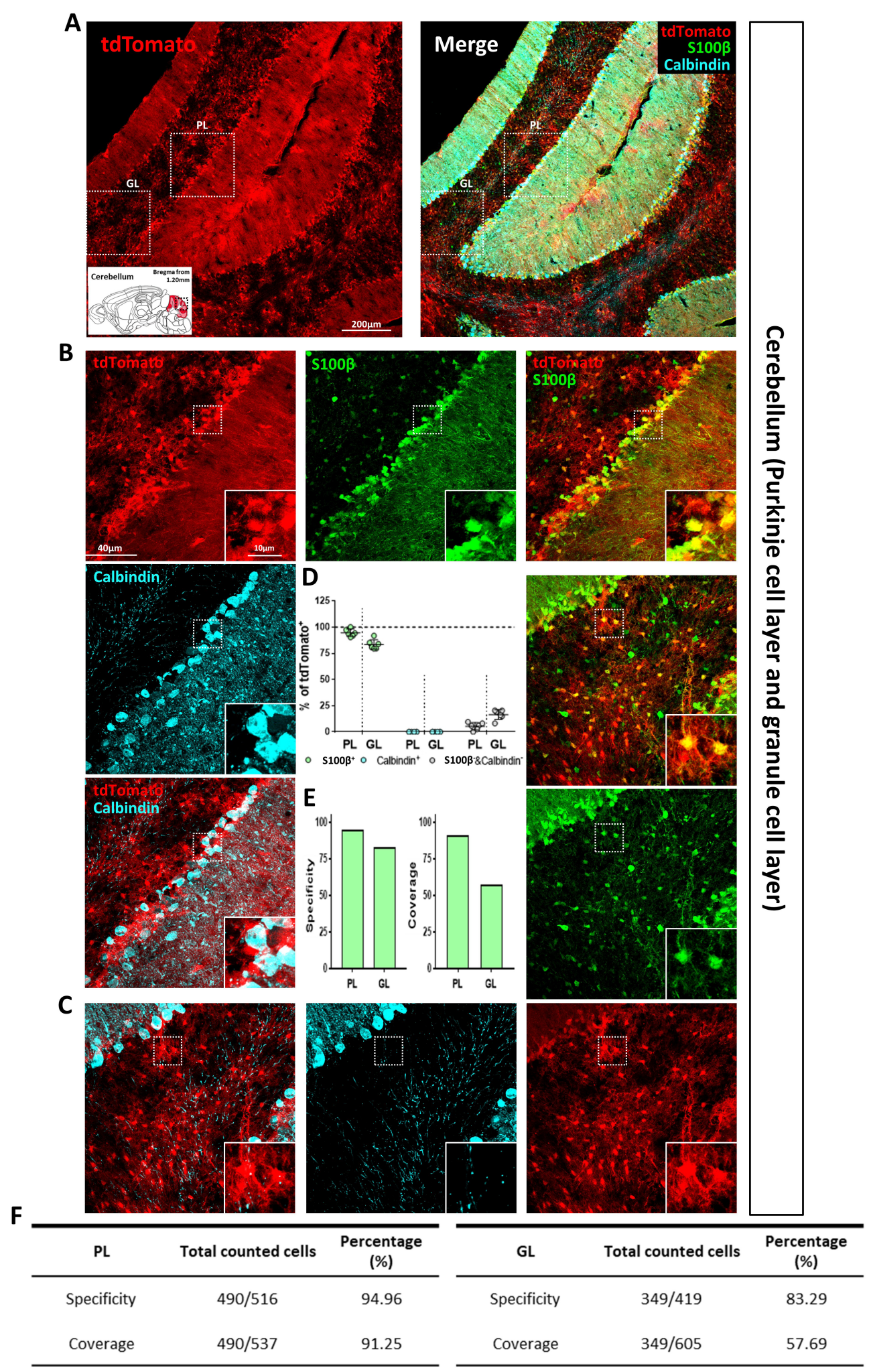

Fig. 7. In cerebellar Purkinje cell layer (PL) and granule cell layer (GL), tdTomato expression driven by hGFAP-CreERT2. (A) Low magnification images of cerebellar PL (left) and GL (right). Each color represents as following description. Green=S100ß; Red=tdTomato; Cyan=Calbindin. (B) High magnification images from the box in PL. (C) High magnification images from the box in GL. (D) Quantification of tdTomato ${ }^{+}$population with S100 $\beta^{+}$(green), Calbindin $^{+}$(cyan) and S100 $\beta$ \&Calbindin (gray) cells in cerebellar PL and GL (Mean \pm SEM, $\mathrm{n}=6$ sections from 3 mice). (E) Summary graph of astrocyte specificity $\left(\right.$ tdTomato $^{+} \& S 100 \beta^{+} /$tdTomato $^{+}$) and coverage (tdTomato $\left.{ }^{+} \& S 100 \beta^{+} / \mathrm{S} 100 \beta^{+}\right)$from each region. (F) Summary table of above analysis $(\mathrm{PL}$ : left, GL: right). 

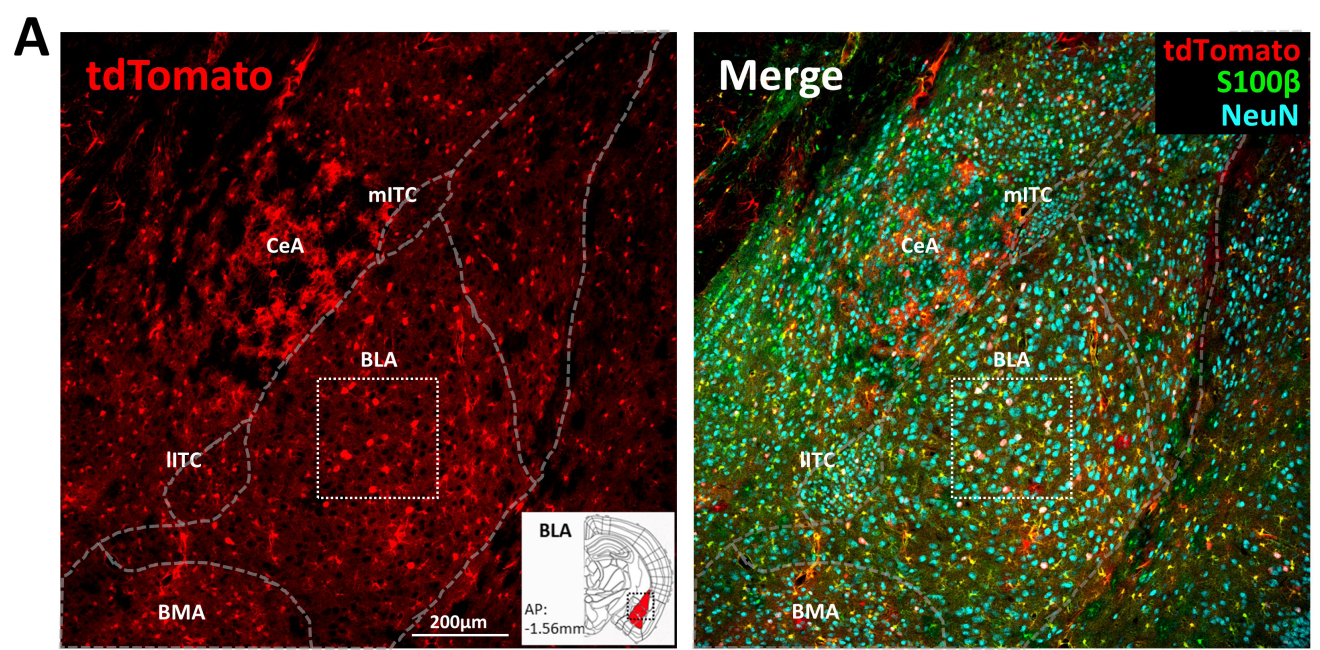

B
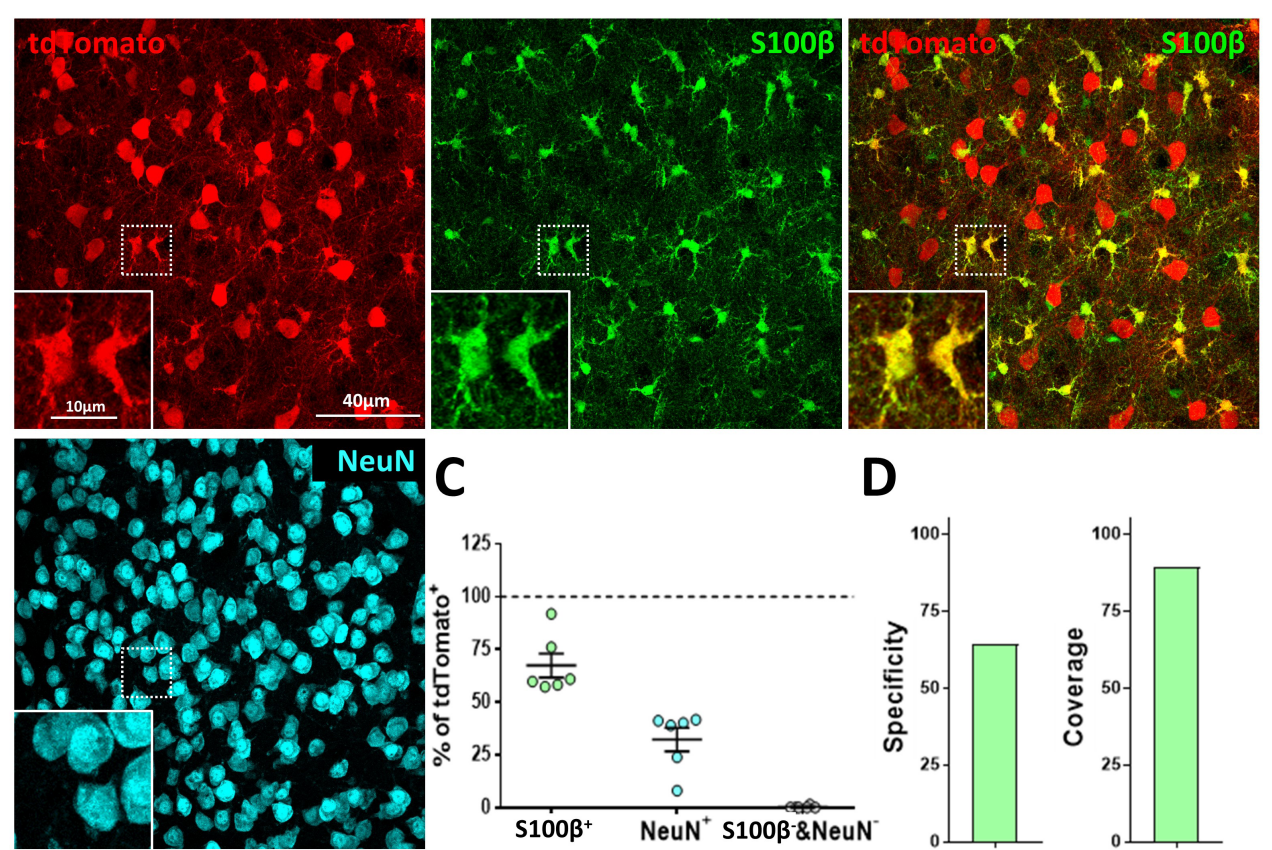

D

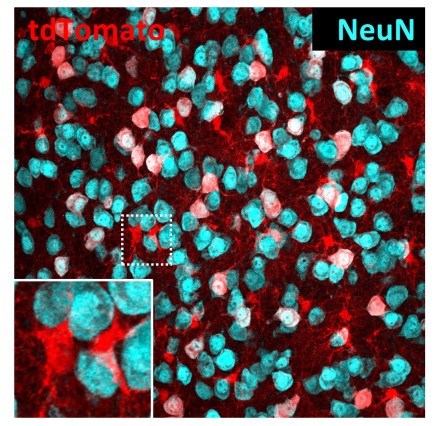

\section{$E$}
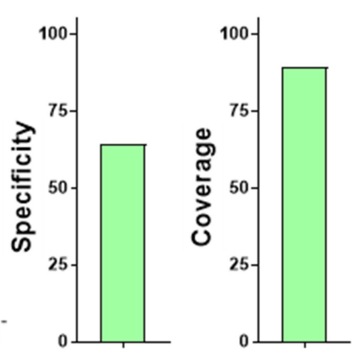

Of tdTomato ${ }^{+}$

Total counted cells Percentage(\%)

Specificity

409/653

64.61

Coverage

$409 / 458$

89.30

Fig. 8. In basolateral amygdala (BLA), tdTomato expression driven by hGFAP-CreERT2. (A) Low magnification images of BLA. Each color represents as following description. Green=S100ß; Red=tdTomato; Cyan=NeuN. (B) High magnification images from the box in (A). (C) Quantification of tdTomato ${ }^{+}$population with $\mathrm{S} 100 \beta^{+}$(green), $\mathrm{NeuN}^{+}$(cyan) and $\mathrm{S} 100 \beta$ \&NeuN (gray) cells in BLA (Mean $\pm \mathrm{SEM}, \mathrm{n}=6$ sections from 3 mice). (D) Summary graph of astrocyte specificity $\left(\right.$ tdTomato $^{+} \& S 100 \beta^{+} /$tdTomato $\left.^{+}\right)$and coverage $\left(\right.$tdTomato $\left.{ }^{+} \& S 100 \beta^{+} / S 100 \beta^{+}\right)$. (E) Summary table of above analysis. 

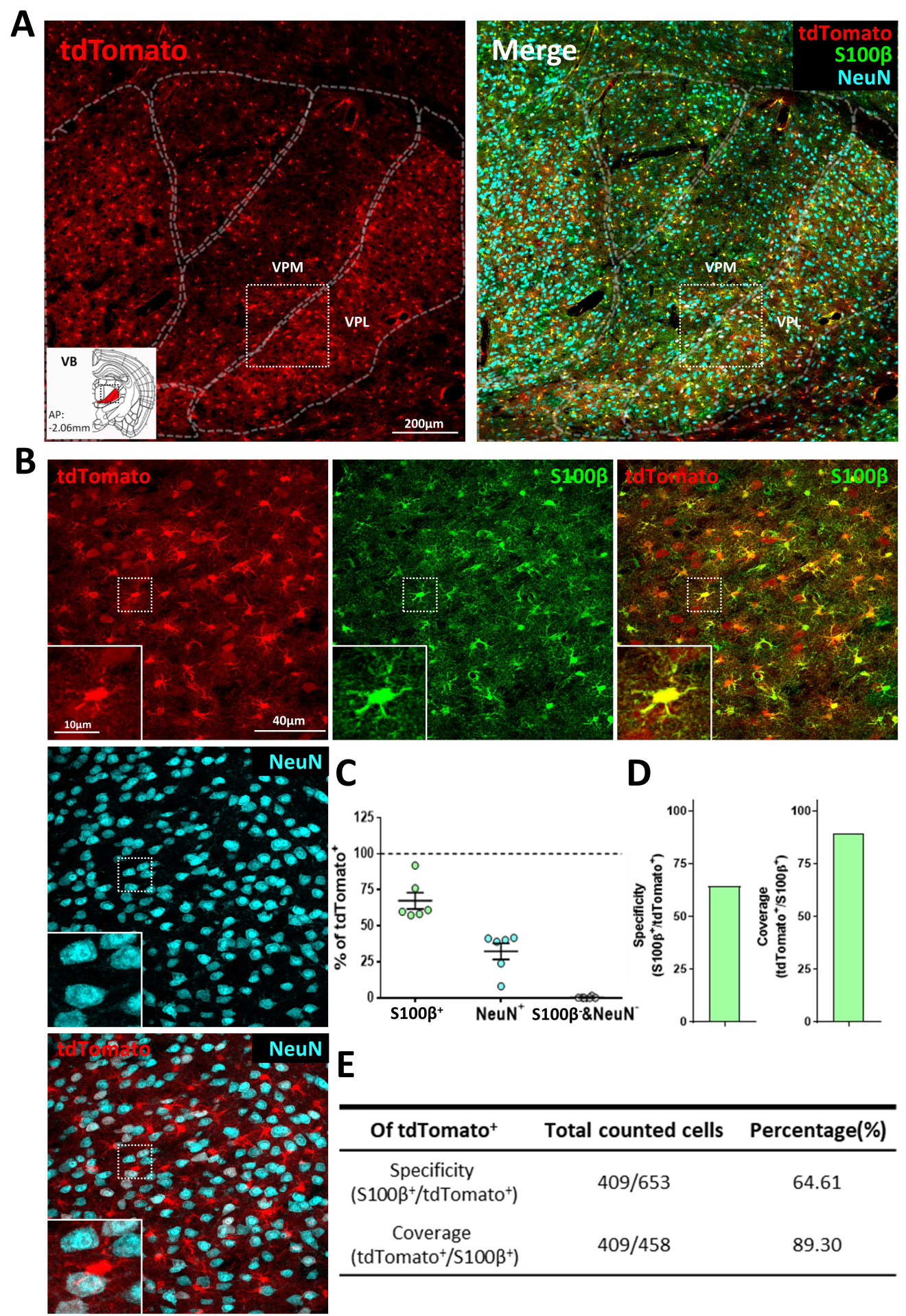

\section{$E$}

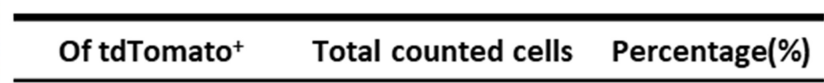

\begin{tabular}{ccc}
\hline $\begin{array}{c}\text { Specificity } \\
\left(\mathrm{S} 100 \beta^{+} / \text {tdTomato }^{+}\right)\end{array}$ & $409 / 653$ & 64.61 \\
$\begin{array}{c}\text { Coverage } \\
\left(\mathrm{tdTomato} / \mathrm{S} 100 \beta^{+}\right)\end{array}$ & $409 / 458$ & 89.30 \\
\hline
\end{tabular}

Fig. 9. In thalamic ventro-basal complex (VB), tdTomato expression driven by hGFAP-CreERT2. (A) Low magnification images of VB. Each color represents as following description. Green=S100ß; Red=tdTomato; Cyan=NeuN. (B) High magnification images from the box in (A). (C) Quantification of tdTomato $^{+}$population with S100 $\beta^{+}$(green), NeuN ${ }^{+}$(cyan) and S100 $\beta$ \&NeuN (gray) cells in VB (Mean \pm SEM, $n=6$ sections from 3 mice). (D) Summary graph of astrocyte specificity $\left(\right.$ tdTomato $^{+} \& S 100 \beta^{+} /$tdTomato $\left.^{+}\right)$and coverage $\left(\operatorname{tdTomato}{ }^{+} \& S 100 \beta^{+} / S 100 \beta^{+}\right)$. (E) Summary table of above analysis. 


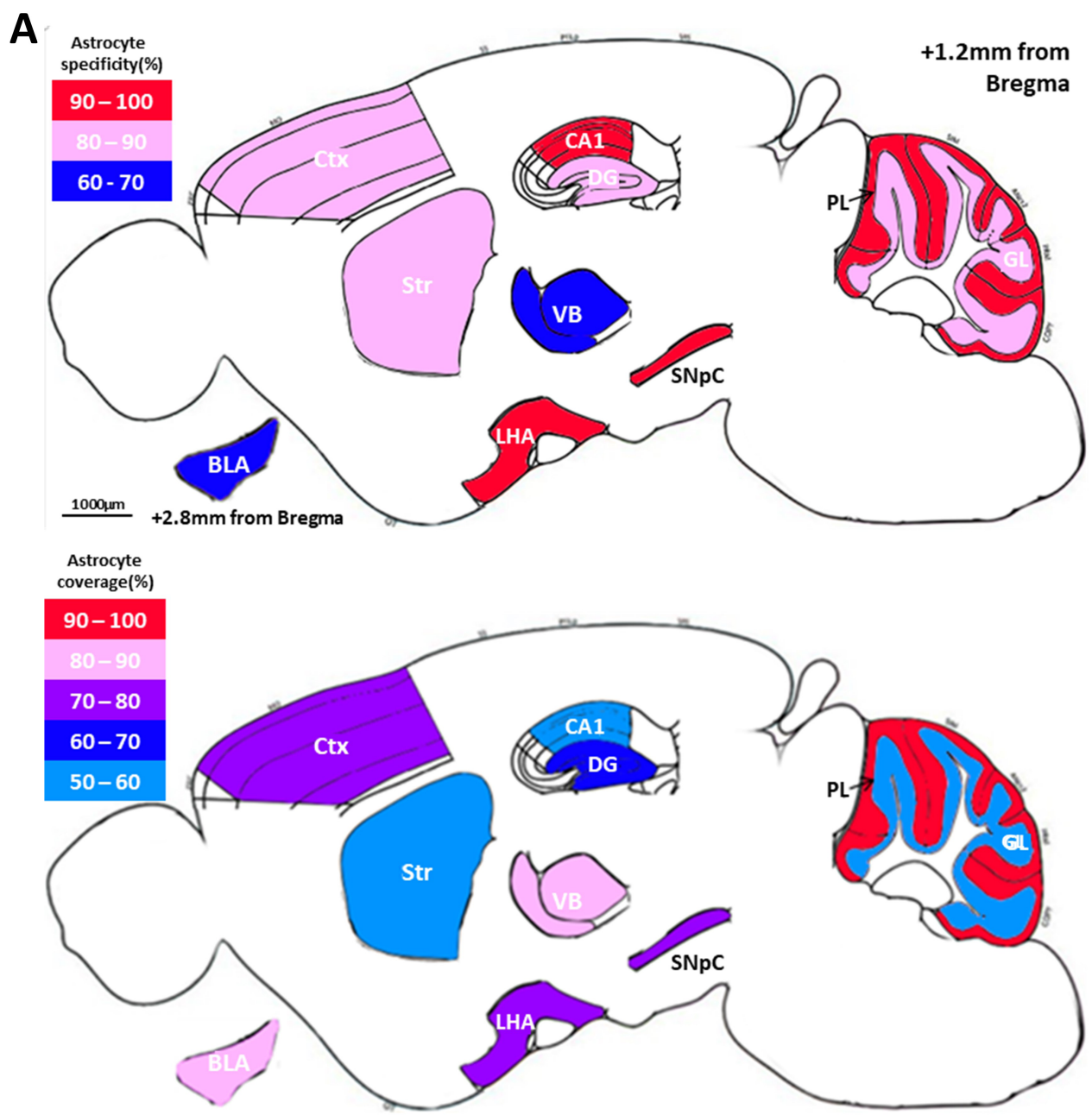

B

\begin{tabular}{|ccc|ccc|}
\hline Region & $\begin{array}{c}{ }^{*} \text { Astrocyte } \\
\text { specificity(\%) }\end{array}$ & $\begin{array}{c}{ }^{* *} \text { Astrocyte } \\
\text { coverage(\%) }\end{array}$ & Region & $\begin{array}{c}\text { Astrocyte } \\
\text { specificity(\%) }\end{array}$ & $\begin{array}{c}\text { Astrocyte } \\
\text { coverage(\%) }\end{array}$ \\
\hline Cortex & 87.69 & 74.07 & SNpC & 97.64 & 72.94 \\
Striatum & 89.02 & 50.69 & Cerebellum & - & - \\
Hippocampus & - & - & Purkinje layer & 94.96 & 91.25 \\
CA1 layer & 92.83 & 56.28 & Granule layer & 83.29 & 57.69 \\
Dentate gyrus & 86.13 & 61.44 & Thalamic VB & 64.61 & 89.30 \\
LHA & 92.20 & 79.24 & BLA & 65.34 & 84.33 \\
\hline
\end{tabular}

${ }^{*}$ Astrocyte specificity: S100 $\beta^{+} \&$ tdTomato ${ }^{+} /$tdTomato $^{+}$

${ }^{* *}$ Astrocyte coverage: $S 100 \beta^{+}$\&tdTomato ${ }^{+} /$S100 $^{+}$

Fig. 10. Summary of astrocyte specificity and coverage of hGFAP-CreERT2 in various brain regions. (A) Schematic sagittal section diagram shows heterogeneous astrocyte specificity (top) and coverage (bottom). Astrocyte specificity is summarized in top diagram, red=over $90 \%$; pink=80 to $90 \%$, and navy $=60$ to $70 \%$. Astrocyte coverage is summarized in bottom diagram, red $=$ over $90 \%$; pink=80 to $90 \%$; purple $=70$ to $80 \%$; navy=60 to $70 \%$; and blue $=50$ to $60 \%$. (B) Summary table of quantitative analysis results in each characterized region.

In cerebellum, hGFAP-CreERT2 mouse shows high astrocyte specificity but differential coverage

In addition, we also scrutinized Cre expression pattern in cerebellar Purkinje cell layer (PL) and granule cell layer (GL). There- fore, we stained with S100 $\beta$ for marking cerebellar astrocyte and Bergmann glia, and with Calbindin for marking Purkinje cell (Fig. 7A). In cerebellar PL, tdTomato expressing cells in each image were highly co-localized with S100 $\beta$ (Fig. 7B and D, green, 94.65 $\pm 1.44 \%$ ) 
but not Calbindin (Fig. 7B and D, cyan, 0.00\%). Therefore, astrocyte specificity was $94.96 \%$ and coverage was $91.25 \%$ (Fig. 8E and F, left).

In GL area (Fig. 7B), tdTomato expressing cells in each image were much more co-localized with S100 $\beta^{+}$cells (Fig. 7C and D, green, $83.46 \pm 1.91 \%$ ) than that of Calbindin ${ }^{+}$cells (Fig. 7C and D, cyan, $0.00 \%)$. Accordingly, astrocyte specificity was $83.29 \%$ and coverage was $57.69 \%$ in GL (Fig. 7E and F, right). These results indicate that hGFAP-CreERT2 mouse shows high astrocyte specificity with over $80 \%$ of specificity in both sub-regions of cerebellum. However, astrocyte coverage is different between these two subregions. Astrocyte coverage is high in PL with over 70\% but low in GL with under $70 \%$.

\section{In BLA and thalamic VB, hGFAP-CreERT2 mouse shows low astrocyte specificity and high coverage}

Finally, we characterized Cre expression in basolateral amygdala (BLA) and thalamic ventro-basal complex (VB) (Fig. 8A and 9A). Even though tdTomato expressing cells in each image were more co-localized with $\mathrm{S} 100 \beta$, the ratio of co-localization with NeuN was also high $\left(\mathrm{S} 100 \beta^{+} \& \operatorname{tdTomato}^{+}\right.$: $66.28 \pm 2.80 \%$, $\mathrm{NeuN}^{+}$\&tdTomato ${ }^{+}: 32.64 \pm 2.70 \%$ ) (Fig. $8 \mathrm{~B}$ and C, S100ß: green, NeuN: cyan). As a result, astrocyte specificity was $65.34 \%$, and coverage was $84.33 \%$ in BLA (Fig. 8D and E). These results indicate that hGFAP-CreERT2 mouse line has low astrocyte specificity with under $80 \%$ of specificity and high astrocyte coverage with over $70 \%$ of coverage in BLA.

Similarly, in thalamic VB, tdTomato expressing cells in each image showed non-specific expression between $\mathrm{S} 100 \beta^{+}$and $\mathrm{NeuN}^{+}\left(\right.$S100 $^{+}$\&tdTomato ${ }^{+}: 67.42 \pm 5.66 \%, \mathrm{NeuN}^{+}$\&tdTomato ${ }^{+}$: $32.33 \pm 5.48 \%$ ) (Fig. 9B and C, S100ß: green, NeuN: cyan). Based on this result, astrocyte specificity was $64.61 \%$, and coverage was $89.30 \%$ in thalamic VB (Fig. 9D and E). These results indicate that hGFAP-CreERT2 mouse line has low astrocyte specificity with under $80 \%$ of specificity and high astrocyte coverage with over $70 \%$ of coverage in thalamic VB.

To sum up, we generated a color-coded brain map to represent the two quantitative aspects; 'astrocyte specificity' and 'astrocyte coverage' (Fig. 10A). Based on this brain map and table, astrocyte specificity of most of investigated regions was over $80 \%$ except in $\mathrm{VB}$ and BLA, whereas astrocyte coverage varied among the various brain regions ranging from $50 \%$ to $90 \%$ (Fig. 10A and B).

\section{DISCUSSION}

Our study quantitatively assessed the astrocyte specificity and coverage of hGFAP-CreERT2 mouse in various brain regions. We have demonstrated that this mouse line expressing Cre under the human GFAP promoter shows differential astrocyte specificity and coverage depending on the brain regions (Fig. 10). Surprisingly, our results are different from those of the previously reported transgenic mouse lines utilizing the same human GFAP promoter $[17,18,21]$. For example, Vaccarino group showed $61.4 \%$ of astrocyte specificity and Baker group showed $52.9 \%$ in cortex. These values are much lower than what we found $(87.69 \%$ in cortex). In addition, Kirchhoff group showed 28\% of astrocyte coverage in cortex $[17,18,21]$, which is far lower than what we found $(74.07 \%$ in cortex). There could be several reasons for this difference. Firstly, the difference of Cre expression properties in each group's mouse line could come from the usage of different Cre reporter lines. Other three groups utilized Z/eg (lacZ/EGFP), ROSA26-EYFP or ROSA26-lacZ, which are the most commonly used Cre reporter lines $[17,18,21]$. However, these reporter lines show much weaker endogenous fluorescence in fixed tissues, compared with the tdTomato in Ai14 [32]. Because of this limitation, additional indirect immunofluorescence staining was needed to validate cell-type specificity for the other reporter lines [33]. In contrast, Ai14 is much more sensitive with far greater fluorescence than other reporter lines and has been proven to be the most optimal reporter line for identification of Cre expressing cells [32]. Thus, it is possible that the difference of Cre reporter mouse line might have caused the discrepancy, even in same brain region and with the same hGFAP promoter.

It is possible that this discrepancy could be due to the difference of mouse strain, too. The mouse lines from Kirchhoff group (FVB/ $\mathrm{N})$ and Baker group $(\mathrm{FVB} / \mathrm{NJ})$ are a different mouse strain from McCarthy group's mice line $(\mathrm{C} 57 \mathrm{BL} / 6 \mathrm{~J})$. A previous report has shown that FVB and C57BL/6N strains have different methylation activities [34]. Therefore, this different methylation condition could differentially regulate the human GFAP promoter activity [35]. Another possibility is that the transgene copy number (TCN) could be the reason for the difference. It has been reported that TCN affects transgene expression level in mammal due to the concatemeric integration [36]. Based on this report, TCN and transgene expression level have inverse correlation [34]. Therefore, if each of these three mouse line's TCN is different with each other, it could explain the difference. However, TCN for each mouse line is unknown. In the future, it would be interesting to determine the exact TCN for each of those mouse lines using the next-generation sequencing (NGS) technology, especially whole genome sequencing (WGS), as a rapid and cost-effective approach [37].

Different tamoxifen administration protocol, including the types of injected ERT2 agonist or dosage of agonist, also can affect tamoxifen-mediated CreERT2 activation [20]. In previous reports, 
authors performed different tamoxifen injection protocol that is different from our study. For example, Vaccarino group used different ERT2 agonist, 4-hydroxytamoxifen (OHT, 33 mg/kg/day for one day) in mouse pups [17]. On the other hand, Baker group used the same ERT2 agonist, tamoxifen, but injected at different dosage of $225 \mathrm{mg} / \mathrm{kg} /$ day for five days [21]. However, Kirchhoff group used the same dosage of tamoxifen as in our study at 100 $\mathrm{mg} / \mathrm{kg} /$ day for five days. Therefore, different tamoxifen administration methods can be a possible reason for the difference between the current study and previous reports.

The heterogeneity of astrocyte specificity in different brain regions, especially the significant neuronal expression in thalamic $\mathrm{VB}$ and BLA, might be caused by alternative gene silencing or heterogeneous activity of human GFAP promoter. It has been well known that histone methylation and acetylation are strongly associated with control of transcriptional activity $[14,34,35]$. If some unknown mechanisms involving histone demethylation or acetylation are differentially involved in thalamic or amygdala neurons, there could be leaky neuronal expression of Cre in those regions. In addition, a differential expression and activity of certain transcriptional factors could contribute to the heterogeneity of both astrocyte specificity and coverage depending on different brain regions [38]. It will be very helpful to elucidate the relationships among the brain regional heterogeneity, expression pattern of transcription factors and epigenetic modification.

Our study provides very useful information regarding the optimal use of the hGFAP-CreERT2 mouse line, depending on the brain region of interest. For example, in cortex, LHA, SNpC and cerebellar Purkinje cell layer, the mouse line shows high astrocyte specificity with over $89 \%$ of specificity and high astrocyte coverage with over $70 \%$ of coverage. In those brain regions, this mouse line would be suitable for genetically modulating astrocytic gene expression in bidirectional ways, either gain-of-function or lossof-function studies. In contrast, in striatum, hippocampus and cerebellar granule cell layer, the mouse line shows high astrocyte specificity with over $80 \%$ of specificity but covering only $50 \sim 60 \%$ of astrocytes. In those brain regions, this mouse line would be more suitable for genetically modulating gene expression in gainof-function studies, rather than loss-of-function studies.

In conclusion, hGFAP-CreERT2 mouse line shows high astrocyte specific Cre expression in most of the brain regions. Our study demonstrates that this transgenic mouse line is very useful for manipulating genes of interest in astrocyte-specific manner, and perhaps is still one of the best genetic tools available for study of astrocyte function in vivo.

\section{ACKNOWLEDGEMENTS}

This research was supported by the Brain Research Program through NRF funded by the Ministry of Science and ICT (2018M3C7A1056682); Institute for Basic Science Grants IBS-R001-D1.

\section{REFERENCES}

1. Kaneko N, Sawada M, Sawamoto K (2017) Mechanisms of neuronal migration in the adult brain. J Neurochem 141:835847.

2. Goubard V, Fino E, Venance L (2011) Contribution of astrocytic glutamate and GABA uptake to corticostriatal information processing. J Physiol 589:2301-2319.

3. Porter JT, McCarthy KD (1997) Astrocytic neurotransmitter receptors in situ and in vivo. Prog Neurobiol 51:439-455.

4. Beltrán-Castillo S, Olivares MJ, Contreras RA, Zúñiga G, Llona I, von Bernhardi R, Eugenín JL (2017) D-serine released by astrocytes in brainstem regulates breathing response to $\mathrm{CO}_{2}$ levels. Nat Commun 8:838.

5. Guthrie PB, Knappenberger J, Segal M, Bennett MV, Charles AC, Kater SB (1999) ATP released from astrocytes mediates glial calcium waves. J Neurosci 19:520-528.

6. Lee S, Yoon BE, Berglund K, Oh SJ, Park H, Shin HS, Augustine GJ, Lee CJ (2010) Channel-mediated tonic GABA release from glia. Science 330:790-796.

7. Woo DH, Han KS, Shim JW, Yoon BE, Kim E, Bae JY, Oh SJ, Hwang EM, Marmorstein AD, Bae YC, Park JY, Lee CJ (2012) TREK- 1 and Best 1 channels mediate fast and slow glutamate release in astrocytes upon GPCR activation. Cell 151:25-40.

8. Kofuji P, Newman EA (2004) Potassium buffering in the central nervous system. Neuroscience 129:1045-1056.

9. Chun H, Lee CJ (2018) Reactive astrocytes in Alzheimer's disease: a double-edged sword. Neurosci Res 126:44-52.

10. Guo Y, Duan W, Li Z, Huang J, Yin Y, Zhang K, Wang Q, Zhang Z, Li C (2010) Decreased GLT-1 and increased SOD1 and HO- 1 expression in astrocytes contribute to lumbar spinal cord vulnerability of SOD1-G93A transgenic mice. FEBS Lett 584:1615-1622.

11. Jo S, Yarishkin O, Hwang YJ, Chun YE, Park M, Woo DH, Bae JY, Kim T, Lee J, Chun H, Park HJ, Lee DY, Hong J, Kim HY, Oh SJ, Park SJ, Lee H, Yoon BE, Kim Y, Jeong Y, Shim I, Bae YC, Cho J, Kowall NW, Ryu H, Hwang E, Kim D, Lee CJ (2014) GABA from reactive astrocytes impairs memory in mouse models of Alzheimer's disease. Nat Med 20:886-896.

12. Foo LC, Dougherty JD (2013) Aldh1L1 is expressed by post- 
natal neural stem cells in vivo. Glia 61:1533-1541.

13. Zhuo L, Theis M, Alvarez-Maya I, Brenner M, Willecke K, Messing A (2001) hGFAP-cre transgenic mice for manipulation of glial and neuronal function in vivo. Genesis 31:85-94.

14. Koh W, Park YM, Lee SE, Lee CJ (2017) AAV-mediated astrocyte-specific gene expression under human ALDH1L1 promoter in mouse thalamus. Exp Neurobiol 26:350-361.

15. Casper KB, McCarthy KD (2006) GFAP-positive progenitor cells produce neurons and oligodendrocytes throughout the CNS. Mol Cell Neurosci 31:676-684.

16. Casper KB, Jones K, McCarthy KD (2007) Characterization of astrocyte-specific conditional knockouts. Genesis 45:292299.

17. Ganat YM, Silbereis J, Cave C, Ngu H, Anderson GM, Ohkubo Y, Ment LR, Vaccarino FM (2006) Early postnatal astroglial cells produce multilineage precursors and neural stem cells in vivo. J Neurosci 26:8609-8621.

18. Hirrlinger PG, Scheller A, Braun C, Hirrlinger J, Kirchhoff F (2006) Temporal control of gene recombination in astrocytes by transgenic expression of the tamoxifen-inducible DNA recombinase variant CreERT2. Glia 54:11-20.

19. Slezak M, Göritz C, Niemiec A, Frisén J, Chambon P, Metzger D, Pfrieger FW (2007) Transgenic mice for conditional gene manipulation in astroglial cells. Glia 55:1565-1576.

20. Srinivasan R, Lu TY, Chai H, Xu J, Huang BS, Golshani P, Coppola G, Khakh BS (2016) New transgenic mouse lines for selectively targeting astrocytes and studying calcium signals in astrocyte processes in situ and in vivo. Neuron 92:11811195.

21. Chow LM, Zhang J, Baker SJ (2008) Inducible Cre recombinase activity in mouse mature astrocytes and adult neural precursor cells. Transgenic Res 17:919-928.

22. Petryszak R, Keays M, Tang YA, Fonseca NA, Barrera E, Burdett T, Füllgrabe A, Fuentes AM, Jupp S, Koskinen S, Mannion O, Huerta L, Megy K, Snow C, Williams E, Barzine M, Hastings E, Weisser H, Wright J, Jaiswal P, Huber W, Choudhary J, Parkinson HE, Brazma A (2016) Expression Atlas update--an integrated database of gene and protein expression in humans, animals and plants. Nucleic Acids Res 44:D746-D752.

23. Park H, Han KS, Seo J, Lee J, Dravid SM, Woo J, Chun H, Cho S, Bae JY, An H, Koh W, Yoon BE, Berlinguer-Palmini R, Mannaioni G, Traynelis SF, Bae YC, Choi SY, Lee CJ (2015) Channel-mediated astrocytic glutamate modulates hippocampal synaptic plasticity by activating postsynaptic NMDA receptors. Mol Brain 8:7.

24. Petr GT, Sun Y, Frederick NM, Zhou Y, Dhamne SC, Hameed MQ, Miranda C, Bedoya EA, Fischer KD, Armsen W, Wang
J, Danbolt NC, Rotenberg A, Aoki CJ, Rosenberg PA (2015) Conditional deletion of the glutamate transporter GLT-1 reveals that astrocytic GLT-1 protects against fatal epilepsy while neuronal GLT-1 contributes significantly to glutamate uptake into synaptosomes. J Neurosci 35:5187-5201.

25. Sun XD, Li L, Liu F, Huang ZH, Bean JC, Jiao HF, Barik A, Kim SM, Wu H, Shen C, Tian Y, Lin TW, Bates R, Sathyamurthy A, Chen YJ, Yin DM, Xiong L, Lin HP, Hu JX, Li BM, Gao TM, Xiong WC, Mei L (2016) Lrp4 in astrocytes modulates glutamatergic transmission. Nat Neurosci 19:1010-1018.

26. Dong HW (2008) The allen reference atlas. A digital color brain atlas of the C57BL/6J male mouse. Wiley, Chichester.

27. Deloulme JC, Raponi E, Gentil BJ, Bertacchi N, Marks A, Labourdette G, Baudier J (2004) Nuclear expression of S100B in oligodendrocyte progenitor cells correlates with differentiation toward the oligodendroglial lineage and modulates oligodendrocytes maturation. Mol Cell Neurosci 27:453-465.

28. Hachem S, Aguirre A, Vives V, Marks A, Gallo V, Legraverend C (2005) Spatial and temporal expression of S100B in cells of oligodendrocyte lineage. Glia 51:81-97.

29. Steiner J, Bernstein HG, Bielau H, Berndt A, Brisch R, Mawrin C, Keilhoff G, Bogerts B (2007) Evidence for a wide extraastrocytic distribution of S100B in human brain. BMC Neurosci 8:2.

30. Kálmán M, Hajós F (1989) Distribution of glial fibrillary acidic protein (GFAP)-immunoreactive astrocytes in the rat brain. I. Forebrain. Exp Brain Res 78:147-163.

31. Doetsch F, Caillé I, Lim DA, García-Verdugo JM, AlvarezBuylla A (1999) Subventricular zone astrocytes are neural stem cells in the adult mammalian brain. Cell 97:703-716.

32. Madisen L, Zwingman TA, Sunkin SM, Oh SW, Zariwala HA, Gu H, Ng LL, Palmiter RD, Hawrylycz MJ, Jones AR, Lein ES, Zeng H (2010) A robust and high-throughput Cre reporting and characterization system for the whole mouse brain. Nat Neurosci 13:133-140.

33. Seymour PA, Sander M (2007) Immunohistochemical detection of beta-galactosidase or green fluorescent protein on tissue sections. Methods Mol Biol 411:13-23.

34. Bai X, Saab AS, Huang W, Hoberg IK, Kirchhoff F, Scheller A (2013) Genetic background affects human glial fibrillary acidic protein promoter activity. PLoS One 8:e66873.

35. Restrepo A, Smith CA, Agnihotri S, Shekarforoush M, Kongkham PN, Seol HJ, Northcott P, Rutka JT (2011) Epigenetic regulation of glial fibrillary acidic protein by DNA methylation in human malignant gliomas. Neuro-oncol 13:42-50.

36. Garrick D, Fiering S, Martin DI, Whitelaw E (1998) Repeatinduced gene silencing in mammals. Nat Genet 18:56-59. 
37. Park D, Park SH, Ban YW, Kim YS, Park KC, Kim NS, Kim JK, Choi IY (2017) A bioinformatics approach for identifying transgene insertion sites using whole genome sequencing data. BMC Biotechnol 17:67.
38. Morel L, Chiang MS, Higashimori H, Shoneye T, Iyer LK, Yelick J, Tai A, Yang Y (2017) Molecular and functional properties of regional astrocytes in the adult brain. J Neurosci 37:8706-8717. 